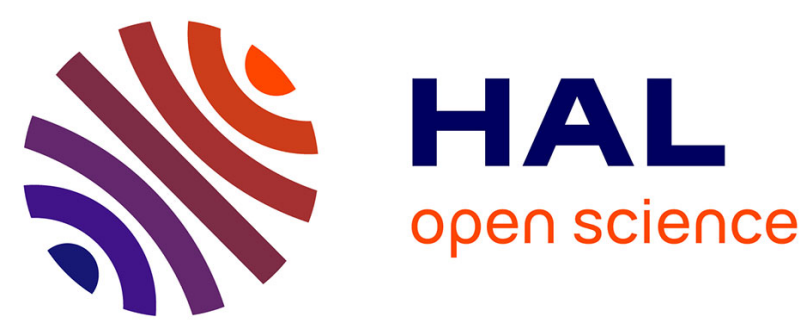

\title{
Fonctionnement hydrique de sols à argile gonflante cultivés. II. - Analyse expérimentale des fonctionnements hydriques associés à deux états structuraux en vertisol irrigué \\ Yves-Marie Cabidoche, Bertrand Ney, N. Jean-Bart, A. Mulcira
}

\section{To cite this version:}

Yves-Marie Cabidoche, Bertrand Ney, N. Jean-Bart, A. Mulcira. Fonctionnement hydrique de sols à argile gonflante cultivés. II. - Analyse expérimentale des fonctionnements hydriques associés à deux états structuraux en vertisol irrigué. Agronomie, 1987, 7 (4), pp.257-270. hal-00884990

\section{HAL Id: hal-00884990 https://hal.science/hal-00884990}

Submitted on 1 Jan 1987

HAL is a multi-disciplinary open access archive for the deposit and dissemination of scientific research documents, whether they are published or not. The documents may come from teaching and research institutions in France or abroad, or from public or private research centers.
L'archive ouverte pluridisciplinaire HAL, est destinée au dépôt et à la diffusion de documents scientifiques de niveau recherche, publiés ou non, émanant des établissements d'enseignement et de recherche français ou étrangers, des laboratoires publics ou privés. 


\title{
Fonctionnement hydrique de sols à argile gon- flante cultivés. II. - Analyse expérimentale des fonctionnements hydriques associés à deux états structuraux en vertisol irrigué
}

\author{
Yves-Marie CABIDOCHE \& Bertrand NEY \\ avec la collaboration technique de N. JEAN-Bart \& A. MULCIBA
}

I.N.R.A., Station d'Agronomie-Science du Sol, Centre de Recherches des Antilles et de la Guyane, B.P. 1232, 97184 Pointe-à-Pître - Cedex, Guadeloupe

Les caractéristiques physiques des vertisols, riches en argile de nature smectitique, sont contraignantes. La proportion de pores de grande taille et leur continuité prend toute son importance pour l'évacuation de l'eau en excès. Certains outils, dont la charrue à disques, conduisent, dans certaines conditions, à obturer ces fentes et à limiter fortement le drainage interne. La volonté d'obtenir une couche travaillée de porosité favorable amène à utiliser le pouvoir de gonflement-retrait des argiles. Soumises à l'action du climat, les mottes se délitent en de fins agrégats. Cependant ceux-ci forment un "mulch » à la surface empêchant le dessèchement, donc l'évolution. des mottes sous-jacentes. Un deuxième passage est généralement pratiqué pour remonter ces mottes à la surface. Par granoclassement, la terre fine, très sèche et peu poreuse, est enfouie sur le fond de labour. La conjonction d'une diminution du drainage interne et de la présence d'agrégats de taille millimétrique, contenant peu d'air et au fort pouvoir gonflant, provoque sous irrigation la formation d'un milieu continu, asphyxiant, limitant fortement le rendement de la culture. Deux états structuraux sont comparés sous différentes pluviométries. Le premier, issu de deux labours successifs effectués au moyen d'une charrue à disques, possède les caractéristiques décrites précédemment. Le second, obtenu par un seul labour de charrue à socs, est caractérisé par une moindre oblitération des fentes, assurant l'évacuation des excès d'eau vers les couches profondes et une transition porale entre couche travaillce et horizons non travaillés moins brutale. La comparaison des comportements d'une culture de tomates montre que la préservation des fissures offre une sécurité vis-à-vis des aléas climatiques et d'une irrigation mal maîtrisée.

Mots clés additionnels : Fonctionnement hydrique, structure, tomate, vertisols irrigation. of irrigated vertisols associated with two structural conditions.

Vertisols were found to have highly constraining physical properties. Infiltration in these soils was largely dependent on cracks. The use of certain ploughing tools, such as the disc plough, led to crack obliteration and decreased infiltration. The swelling and shrinking characteristics of smectic clay, often used to obtain fine tilth were investigated. Clods cracked and gave rise to fine earth on the surface. Repetitive passages were necessary to ensure dehydration of the clods protected by the mulch. These passages covered the plough pan with very dry poorly porous fine aggregates. After irrigation, the presence of a perched water table on the plough pan led to saturation of the soil up from the base of the ploughed layer. Rehydration of the fine earth material gave rise to a horizon with a massive soil structure. Two structural soil conditions, obtained by two plough types (disc and cart), were compared under a high irrigation level. Crack obliteration and water accumulation above the plough pan led to a drop in tomato yield, while crack preservation maintained yields at high irrigation levels.

Additional key words : Hydric behaviour, structure, tomato, vertisol irrigation. 


\section{INTRODUCTION}

Les vertisols représentent plus de la moitié des surfaces cultivables des toposéquences de sols à argile gonflante sur calcaires coralliens, dont ils occupent les parties concaves ou les bas de versants.

L'analyse préliminaire du fonctionnement hydrique de ces sols à argiles gonflantes, sous des cultures de tomates du périmètre irrigué de Grande-Terre de Guadeloupe, a montré l'apparition fréquente d'un excès d'eau dans les vertisols profonds (NEY, 1986). Les états hydriques observés sous irrigation excédentaire suggèrent le rôle d'une oblitération de la porosité de drainage fissurale, au fond de la couche travaillée, dans l'apparition de l'excès d'eau. Cette oblitération est initiée par un lissage du fond de labour consécutif à 2 passages de charrue à disques; elle semble renforcée par l'interposition, entre la couche travaillée et les fissures, d'un niveau très humide et de structure continue, qui s'installe à la suite du regonflement des agrégats de la base de la couche travaillée initialement très secs.

L'objectif du présent article est de montrer expérimentalement, grâce à des contrôles directs de la porosité et de son occupation par l'air et l'eau, et par une caractérisation des transferts d'eau sous différents régimes d'irrigation, le rôle de différents fonds de labour et de différentes structures de la couche travaillée dans l'apparition de niveaux saturés en vertisols irrigués. Le suivi de la réponse d'une culture de tomate, espèce particulièrement sensible (DUTHION, 1973 ; Perigaud, 1967), permet de révéler l'excès d'eau qui peut alors s'installer.

\section{MATÉRIEL ET MÉTHODES}

\section{A. Dispositif et obtention des traitements}

L'expérimentation a été conduite à la ferme I.N.R.A. de May (St. François, Guadeloupe), sur des vertisols calciques contenant plus de 70 p. 100 de smectite dans la terre totale.

Deux parcelles de $25 \times 25 \mathrm{~m}$, distantes de $50 \mathrm{~m}$ en ont constitué le support. Une cartographie de la résistivité (CABIDOCHE, 1986) a permis de contrôler l'épaisseur de l'argile, toujours supérieure à $2 \mathrm{~m}$.

Pour obtenir les états du sol voulus, 2 types de préparation sont appliqués, chacun à la moitié des 2 parcelles :

- l'oblitération des fentes de retrait par lissage et la couche travaillée constituée de fins agrégats sont obtenus après 2 passages consécutifs de charrue à disques (traitement $\mathrm{CD}$ ) comme observé précédemment. Ces 2 passages ont lieu les 15 février et 5 mars 1984 ;

- la préservation de la continuité de la majorité des fissures et une couche travaillée constituée de mottes plus grossières proviennent d'un unique passage de charrue à socs (traitement CS), le 20 février.

Après un sillonnage général, à $1,6 \mathrm{~m}$ d'écartement, le 20 mars, la plantation a eu lieu le 23 mars, tous les $0,30 \mathrm{~m}$ (20 000 plants/ha).

L'irrigation a dû être appliquée par aspersion à poste fixe (sprinklers). Devant l'impossibilité de réali- ser une couverture homogène avec ce type de matériel, nous avons été amenés à définir $a$ posteriori les traitements d'irrigation grâce à une caractérisation de la structure spatiale de l'hétérogénéité au moyen d'un maillage de pluviomètres; cette structure est fidèle d'une date à l'autre, grâce à une stabilité de la direction du vent (alizé d'est) et de son diagramme journalier de vitesse.

Les régimes d'irrigation se différencient au-delà du $20^{\mathrm{e}} \mathrm{j}$ par les doses (et non les fréquences) : irrigation rationnée à égale aux besoins dans la parcelle Est, égale aux besoins à excédentaire dans la parcelle Ouest (fig. 1). Les traitements d'irrigation sont définis comme les classes d'apport total du 23 mars au 8 juin
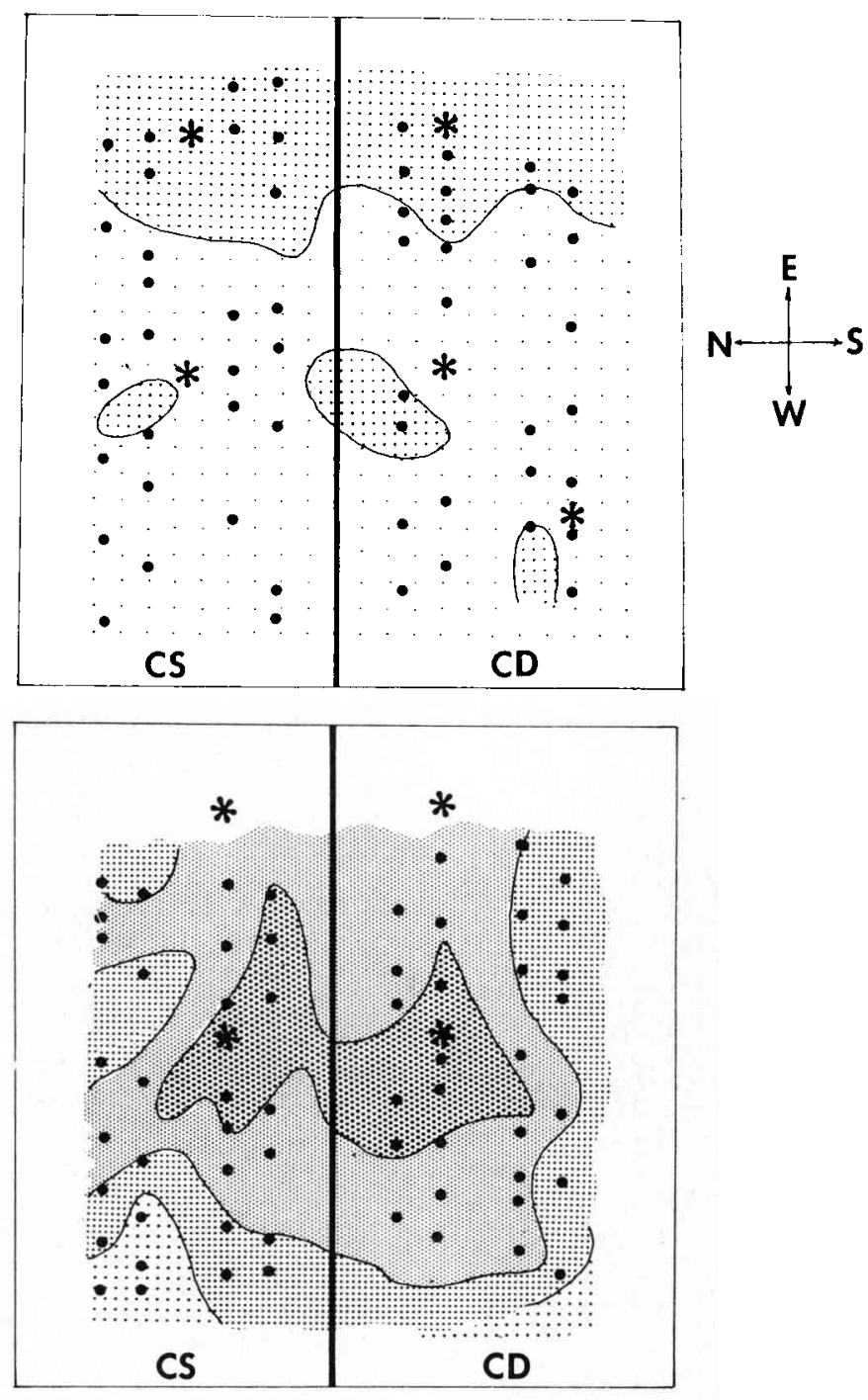

$0 \quad 5 \quad 10 \quad 15 \quad 20 \mathrm{~m}$

Figure 1

Cartographie de la pluviométrie pendant la durée du cycle sur les 2 parcelles.

Rainfall map on the two plots.

$\square 300<P<399 \mathrm{~mm}$ (traitement 11)

$400<P<499 \mathrm{~mm}$ (traitement 12)

$500<P<599 \mathrm{~mm}$ (traitement 13)

$600<P<699 \mathrm{~mm}$ (traitement 14)

[.].] $700<P<800 \mathrm{~mm}$ (traitement I5)

* Position des arroseurs. Sprinkler position.

- Position des plantes suivies. Plant position. 
(date de floraison des 10 premiers bouquets) : 300 à $400 \mathrm{~mm}$ pour I1, de 400 à $500 \mathrm{~mm}$ pour I2, de 500 à $60 \mathrm{~mm}$ pour $\mathrm{I} 3$, de 600 à $700 \mathrm{~mm}$ pour I4, de 700 à $800 \mathrm{~mm}$ pour I5. Les régimes extrêmes, I1 et I5 sont mentionnés figure 2 .
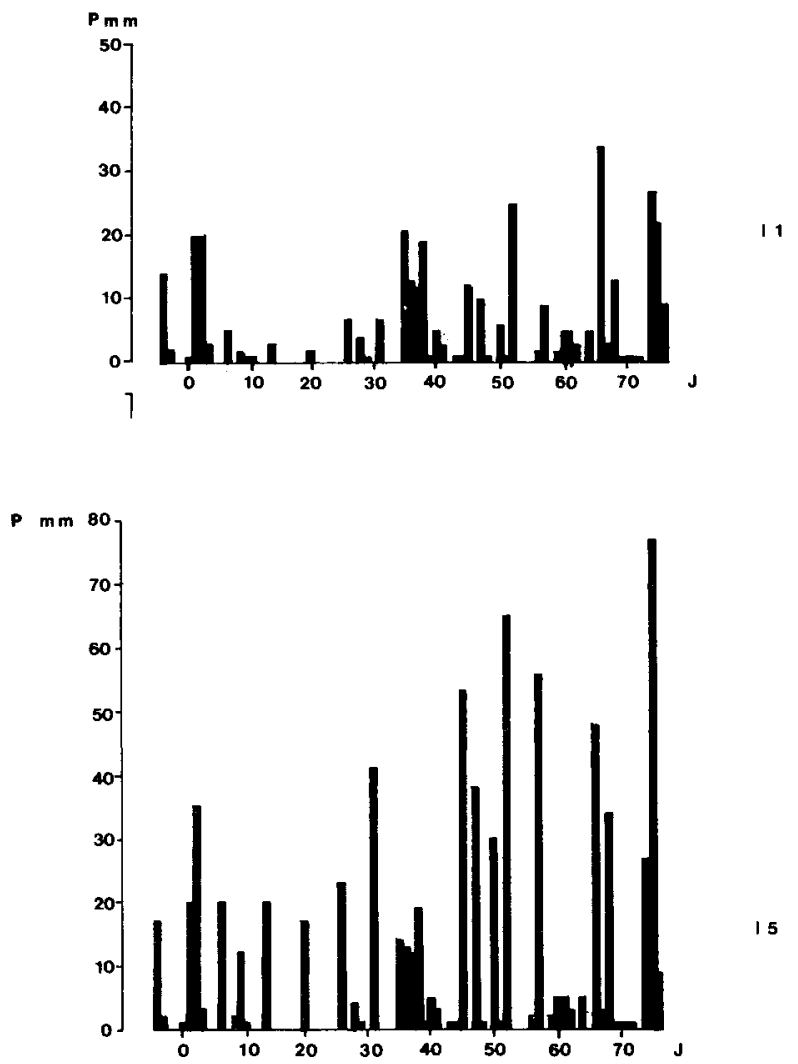

Figure 2

Pluviométrie moyenne $(P)$ des traitements d'irrigation extrêmes II et I5.

Average rainfall $(P)$ of irrigation treatment $I I$ and 15 .

$J$ Jours après plantation. Days after planting.

L'ETP, calculée à partir du rayonnement global selon SCHOCH et al. (1975), est relativement constante (entre 4 et $6 \mathrm{~mm}$ par j) et égale à $480 \mathrm{~mm}$ pendant cette période.

La variété de tomate choisie est à port indéterminé (Lycopersicon esculentum $\mathrm{cv}$. tropic). La fertilisation (300 uN, $240 \mathrm{uP}, 370 \mathrm{uK}$ ) a été fractionnée en 2 apports (plantation et floraison). Les plantes ont été taillées sur 2 bras et tuteurées 3 semaines après la plantation. Les interrangs ont été désherbés par plusieurs passages de fraise et épandages de paraquat, en alternance ; les rangs ont été désherbés manuellement. Les plantes suivies sont au nombre de 120 , réparties au hasard sur les 2 parcelles. Pour chacune d'elle est calculée une pluviométrie reçue, par interpolation linéaire à partir des données des pluviomètres les plus proches (fig. 1).

\section{B. Méthodes}

L'évolution de la balance air/eau dans le sol est confrontée à la réponse d'un peuplement végétal sensible au défaut d'aération du sol.

\section{Suivi des porosités du sol et de leur occupation par les phases liquide et gazeuse}

Les grandeurs de diagnostic retenues pour caractériser l'état poral sont les volumes spécifiques d'air et d'eau, obtenus à différentes profondeurs du sol soumis, à l'action des outils et du climat, puis à différentes doses d'irrigation.

Le volume spécifique d'eau $\left(\nu_{W}, \mathrm{~cm}^{3} \cdot \mathrm{g}^{-1}\right)$ est assimilé en valeur à la teneur en eau pondérale $\left(W, g \cdot g^{-1}\right)$, la précision des mesures permettant de négliger l'intervention de la masse volumique de l'eau.

Le volume spécifique d'air $\left(v_{\mathrm{a}}, \mathrm{cm}^{3} \cdot \mathrm{g}^{-1}\right)$ est calculé selon la formule suivante :

$$
\begin{aligned}
v_{\mathrm{a}}= & \left(1 / \gamma_{\mathrm{d}}\right)-\left(1 / \gamma_{\mathrm{s}}^{\prime}\right)-v_{\mathrm{W}} \\
\gamma_{\mathrm{d}}= & \text { masse volumique sèche }\left(\mathrm{g} \cdot \mathrm{cm}^{-3}\right) \\
\gamma_{\mathrm{s}}^{\prime}= & \text { masse volumique de solide avec air piégé } \\
& \left(2,16 \mathrm{~g} \cdot \mathrm{cm}^{-3}\right) .
\end{aligned}
$$

L'appréhension de ces grandeurs nécessite plusieurs niveaux de perception. Le contrôle de l'augmentation de porosité consécutive à un travail du sol ne peut se faire par de simples mesures de masse volumique dans les sols à argile gonflante : la porosité intra-agrégats n'est pas stable, à cause des mouvements de solide consécutifs aux humectations et dessiccations. Le volume poral additionnel, consécutif à la structuration obtenue par le labour, sera donc calculé par différence entre le volume poral total et le volume poral intraagrégats qu'il convient de mesurer. Cette démarche conduit à effectuer des mesures de volumes d'échantillons à différents niveaux : celui de l'agrégat (de petite taille, indivisible sans perturbation de sa géométrie), celui de la motte (assemblage d'agrégats de cohésion interne nettement supérieure à sa cohésion avec l'environnement), celui enfin de la couche travaillée dans sa globalité.

Les volumes des agrégats sont évalués par immersion dans le mercure, ceux des mottes par la poussée d'Archimède dans l'eau, après ensachage sous vide dans un film PVC. Cette dernière méthode est beaucoup plus rapide que celle utilisant l'enrobage à la paraffine ; elle permet également de mesurer la teneur en eau de la totalité de l'échantillon, celle-ci étant d'autant plus hétérogène que l'échantillon est de grande taille. La comparaison des 2 méthodes révèle des différences relatives de 1 à 3 p. 100, par excès pour l'ensachage : ce dernier prend en compte les macropores fissuraux ou tubulaires que peut envahir partiellement la paraffine. Ces mesures sont effectuées du premier passage de charrue à la plantation.

Les volumes spécifiques globaux de la couche travaillée sont également mesurés du début de la préparation du sol à la plantation, mais d'une manière indirecte. L'utilisation du densitomètre à membrane pour la détermination des volumes totaux est impossible durant cette période, d'une part à cause de l'hétérogénéité et de la grande taille des mottes, et d'autre part à cause de la dessiccation prononcée, qui, disjoignant les agrégats, rend inévitable les déformations du « moule» de l'échantillon prélevé. Une méthode de mesure topographique du volume a donc été mise en œuvre.

L'état initial, avant travail du sol, est défini par la position des 30 premiers centimètres, destinés à être travaillés, sur une représentation de Haynes · cette 
position est obtenue grâce à des mesures de teneur en eau et de masse volumique $\left(\gamma_{\mathrm{d}_{\mathrm{e}}}\right)$ au densitomètre à membrane.

Le soufflage de la couche travaillée est calculé avant plantation, à partir d'une comparaison des sections topographiées de la couche travaillée, à cette date, et à l'état initial, selon les principes suivants :

- en l'absence d'extraction racinaire de l'eau, et compte tenu du degré de dessiccation en surface, les horizons non travaillés sont peu ou pas desséchés (RITCHIE \& BURNETT, 1971) ; la cote du fond de labour n'a donc pas changé, seule la cote de la surface varie ;

- les labours sont unidirectionnels E-W ; entre l'évolution maximale du dessèchement de la couche travaillée avant plantation (ep) et l'état initial (ei), le rapport des sections verticales $(\mathrm{S})$, de direction $\mathrm{N}-\mathrm{S}$, de la couche travaillée, est égal au rapport des volumes (V) :

$$
\mathrm{V}_{\mathrm{ep}} / \mathrm{V}_{\mathrm{ei}}=\mathrm{S}_{\mathrm{en}} / \mathrm{S}_{\mathrm{ei}}
$$

- le solide se conservant, on peut calculer la masse volumique globale de la couche travaillée à la plantation $\left(\gamma_{\mathrm{d}_{\mathrm{ep}}}\right)$ :

$$
\left(1 / \gamma_{d_{e p}}\right)=\left(S_{e p} / S_{e i}\right) \cdot 1 / \gamma_{d_{e i}} ;
$$

- la mesure des teneurs en eau $\left(W_{\mathrm{ep}}\right)$ par une coupe hydrique, permet d'obtenir les volumes spécifiques d'eau $\left(v_{w_{e p}}\right)$ et d'air $\left(v_{a_{e p}}\right)$ de la couche travaillée, à la plantation :

$$
\begin{aligned}
& v_{w_{e p}}=W_{e p} / 1 \\
& v_{a_{e p}}=\left(\left(S_{e p} / S_{e i}\right) \cdot 1 / \gamma_{d_{e i}}\right)-\left(1 / \gamma_{s}^{\prime}\right)-v_{w_{e p}}
\end{aligned}
$$

- dans la pratique, compte tenu de la dispersion des masses volumiques à l'état initial, et des teneurs en eau de la couche travaillée à la date d'évolution maximale, nous retiendrons simplement un bornage de ces volumes spécifiques à la plantation. En tout état de cause, les minorants des volumes spécifiques d'air seront suffisants pour les interprétations qui vont suivre.

Les volumes spécifiques des horizons de la couche travaillée sont mesurés en cours de culture. Dès les premières irrigations, les horizons de la couche travaillée se présentent comme latéralement homogènes vis-à-vis d'un volume de $1 \mathrm{dm}^{3}$, en même temps que diminuent la cohésion et l'individualité des mottes ; le densitomètre à membrane est donc à nouveau utilisable pour déterminer les volumes spécifiques des phases des différents horizons de la couche travaillée, en cours de culture.

\section{Estimation qualitative des transferts d'eau dans le sol, sous irrigation}

Cette estimation est réalisée à partir d'une caractérisation qualitative des stockages d'eau par la méthode smectométrique (CABIDOCHE et al., 1986) qui permet de contrôler les champs de mouvements verticaux du sol grâce à des jalons vissés à différentes profondeurs. Cette méthode est utilisée ici pour vérifier la fonctionnalité des fentes de retrait dans les transferts rapides d'eau : si elles sont fonctionnelles, le gonflement, et donc la montée des jalons, se propage en remontant. Dans le cas contraire, la lente diffusion provoque le gonflement des horizons du haut vers le bas à partir du fond de la couche travaillée.
Quatre batteries, constituées chacune par 5 jalons vissés à $0,4,0,6,0,8$ et $1,2 \mathrm{~m}$ de profondeur lors de la pose, sont placées dans les traitements extrêmes (CD et CS 12 et 15). La cote des jalons est suivie au niveau de chantier et à la mire millimétrique, en référence à un jalon fixe ancré dans le calcaire ; l'incertitude absolue est de $\pm 1 \mathrm{~mm}$.

Soit $\mathrm{Zx}(\mathrm{t})$ la profondeur à l'instant $\mathrm{t}$, de l'extrémité d'un jalon placé initialement à la profondeur $\mathrm{x}$. Le gonflement global $(G)$, entre 2 dates $i$ et $i+1$, de la couche limitée par les prutondeurs $x_{j}$ et $x_{j+1}$ sera :

$$
G=\Delta_{t=i}^{i+1} Z_{j+1}(t)-\Delta_{i=i}^{i+1}{Z x_{j}}_{j}(t) .
$$

Si les couches sont d'épaisseurs inégales, on calculera le gonflement élémentaire $(\mathrm{g})$, grandeur adimensionnelle :

$$
\mathrm{g}=\mathrm{G} /\left(\mathrm{x}_{\mathrm{j}+1}-\mathrm{x}_{\mathrm{j}}\right)
$$

\section{Mesures sur la culture, révélatrice des états du milieu}

Le paramètre suivi est le nombre de fleurs nouées produites. Celui-ci est le produit du nombre de fleurs formées par le pourcentage de nouaison, tous deux fortement influencés par le niveau d'aération du sol (FloCKER \& MENARY, 1960 ; WIEBE, 1949).

Afin d'obtenir une évolution continue de ce paramètre, permettant de dater avec précision, l'apparition du déséquilibre air/eau, le port a été choisi indéterminé. On considèrera que la date d'apparition des effets des excès d'eau sur le niveau d'aération du sol débute lorsque les moyennes du nombre cumulé de fleurs nouées sont significativement différentes entre les traitements d'irrigation.

Le rendement final a été calculé en nombre de fruits total produit par chaque plante. Ce nombre est très fortement corrélé au rendement pondéral final (CAVICCHI \& SilveTTI, 1976 ; SingH \& SiNGH, 1980). Le rendement de chaque plante est comparé avec la dose d'irrigation, calculée par interpolation, qu'elle a reçue.

Les enregistrements ont porté sur 120 plantes suivies du 17 avril, date de floraison de la première plante (25 $\mathrm{j}$ après plantation), au 8 juin $(77 \mathrm{j}$ après plantation), aucun fruit commercialisable n'étant apparu après cette date.

Des profils culturaux ont été ouverts à la floraison et en fin de culture, afin de décrire l'état sanitaire et l'extension du système racinaire.

\section{RÉSULTATS}

\section{A. Caractérisation des états du milieu}

\section{Etat initial (ei)}

On constate sur les profils ouverts avant le passage des outils, l'existence de fissures réparties selon un maillage d'ordre de grandeur métrique. Deux horizons sont nettement différenciables. Les 30 premiers centimètres, gris sombre, possèdent une importante porosité tubulaire due à l'activité biologique intense ; cette porosité, très élevée en surface, s'atténue vers le bas (tabl. 1), notamment pour l'air. La teneur en eau est relativement homogène $\left(0,25\right.$ à $\left.0,30 \mathrm{~g} \cdot \mathrm{g}^{-1}\right)$. En dessous, se développe un horizon jaune, de méso-structure 
TABLEAU 1

Volumes spécifiques de sol sec $\left(1 / \gamma_{d}\right)$, d'eau $\left(v_{w}\right)$ et d'air $\left(v_{a}\right)$ avant le passage des outils (ei).

$\operatorname{Dry}$ soil $\left(1 / \gamma_{d}\right)$, water $\left(v_{w}\right)$ and air $\left(v_{a}\right)$ specific volumes before planting (ei).

\begin{tabular}{cccc}
\hline \hline Profondeur (cm) & $1 / \gamma_{\mathrm{d}}\left(\mathrm{cm}^{3} \cdot \mathrm{g}^{-1}\right)$ & $v_{\mathrm{w}_{\mathrm{ei}}}\left(\mathrm{cm}^{3} \cdot \mathrm{g}^{-1}\right)$ & $v_{\mathrm{a}_{\mathrm{ei}}}\left(\mathrm{cm}^{3} \cdot \mathrm{g}^{-1}\right)$ \\
\hline \multirow{2}{*}{$0-5$} & 1,15 & 0,46 & 0,32 \\
& 1,15 & 0,40 & 0,29 \\
\hline \multirow{2}{*}{$10-15$} & 1,03 & 0,42 & 0,15 \\
& 0,98 & 0,41 & 0,11 \\
\hline \multirow{2}{*}{$20-30$} & 0,94 & 0,44 & 0,03 \\
& 0,97 & 0,45 & 0,06 \\
& 0,98 & 0,45 & 0,07 \\
\hline \hline
\end{tabular}

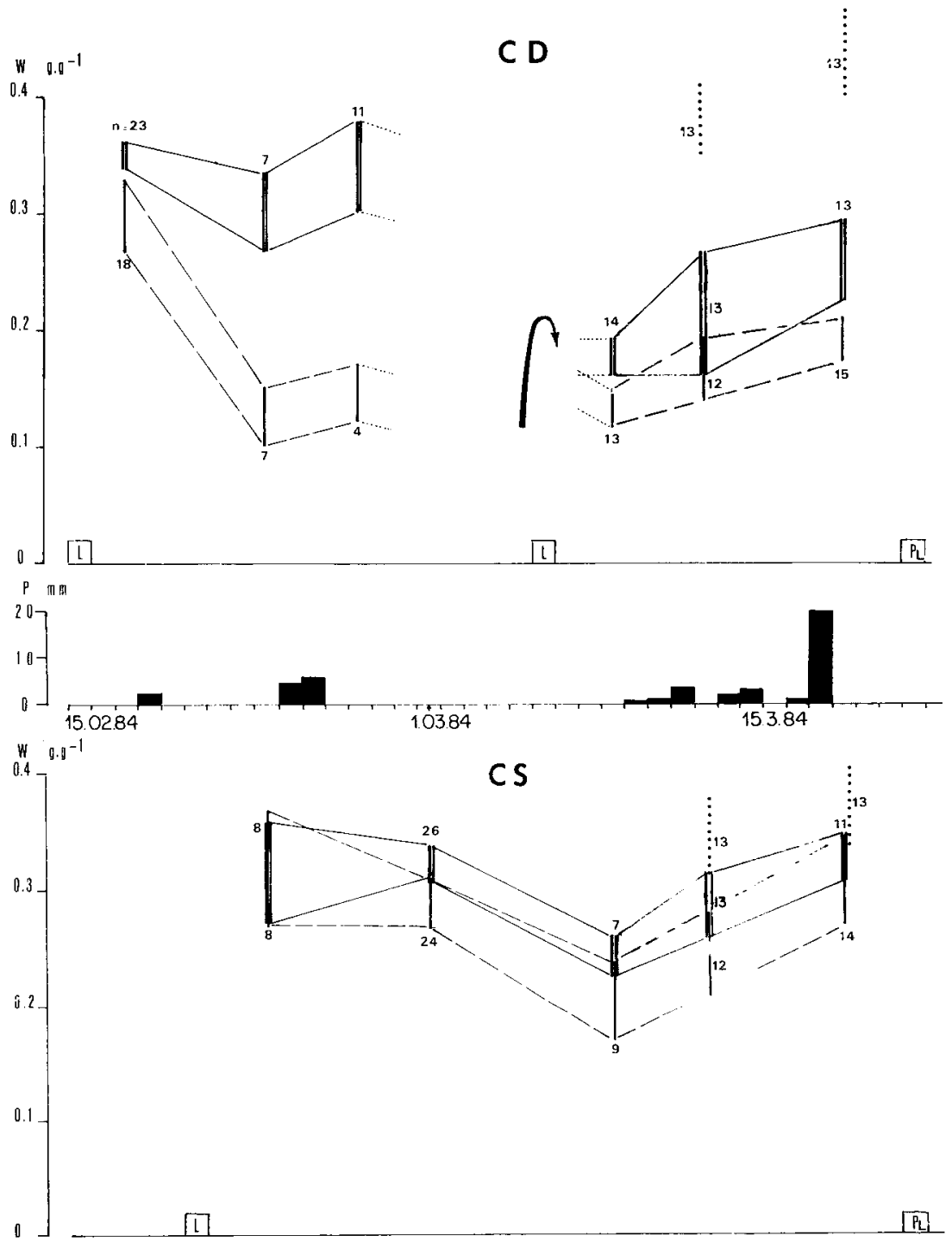

continue, à rares pores tubulaires. L'aération y est quasiment nulle comme dans les derniers centimètres de l'horizon précédent (hors fissures) et la teneur en eau beaucoup plus hétérogène $\left(0,35\right.$ à $\left.0,50 \mathrm{~g} \cdot \mathrm{g}^{-1}\right)$.

\section{Etat avant la plantation (ep)}

a) Evolution de l'état hydrique sous l'action du climat entre labour et plantation (fig. 3)

Dans le traitement CD, seules les mottes de surface $(0-15 \mathrm{~cm})$ montrent une dessiccation prononcée. Il faut attendre le retournement du deuxième labour pour constater un dessèchement de l'ensemble de la couche travaillée $\left(0,12\right.$ à $0,20 \mathrm{~g} . \mathrm{g}^{-1}$ le 9.03 .84$)$. Après une petite période pluvieuse, la réhumectation concerne surtout le sommet de la couche non travaillée : il s'ins-

Figure 3

Evolution des teneurs en eau (W) des mottes sous l'action des outils et du climat.

Effects of tools used and of climate on water content $(W)$ of clods.

Intervalle de confiance à $95 \mathrm{p}$. 100. Confidence interval $95 \%$ :

(Couche $5-15 \mathrm{~cm}$ de l'horizon travaillé.

$35-15 \mathrm{~cm}$ depth of the tilled layer.

(Couche $20-30 \mathrm{~cm}$ de l'horizon travaillé.

$320-30 \mathrm{~cm}$ depth of the tilled layer.
: $\{$ Horizon non travaillé. Untilled layer.

P Pluviométrie. Rainfall.

L Labour. Tillage.

Pl Plantation. Planting.

$n$ Effectif de l'échantillon. Clod number in sample 
talle alors un très fort gradient croissant d'humidité entre les couches travaillées et non travaillées.

Dans le traitement CS, l'ensemble de la couche travaillée est soumise à la dessiccation, qui demeure plus faible qu'en $C D\left(0,17\right.$ à $0,27 \mathrm{~g} \cdot \mathrm{g}^{-1}$ le 9.03.84). La réhumectation concerne de la même façon les couches travaillée et non travaillée ; le gradient vertical d'humidité reste faible.

\section{b) Etat poral avant la plantation}

Des profils culturaux ouverts peu avant la plantation permettent de vérifier l'adéquation des états structuraux aux « états-objectifs » recherchés. Les fentes sont oblitérées et le fond de labour lissé en $C D$ et non en CS. Pour le traitement CD, on obtient dans la couche travaillée un assemblage d'agrégats polyédriques individualisés, très secs et de petite taille en surface et sur le fond de labour. Dans la partie intermédiaire, se trouvent des mottes centi- à décimétriques. En CS, la première catégorie d'éléments structuraux ne se rencontre qu'en surface. La géométrie des mottes grossières sous-jacentes a peu évolué.

La mesure des volumes spécifiques d'eau et d'air internes des mottes de 100 à $600 \mathrm{~cm}^{3}$ ) montre dans les 2 cas une balance analogue entre l'air et l'eau (fig. 4). Cependant, l'humidité étant plus faible en $C D$, les volumes spécifiques d'air sont plus faibles en valeur absolue qu'en CS. Quant aux agrégats plus fins du traitement $C D$, prélevés au-dessus du fond de labour, ils ont un volume spécifique d'air nul (leur volume spécifique d'eau supérieur est dû à l'arrière effet d'une petite pluie) ; l'émiettement de la couche travaillée CD

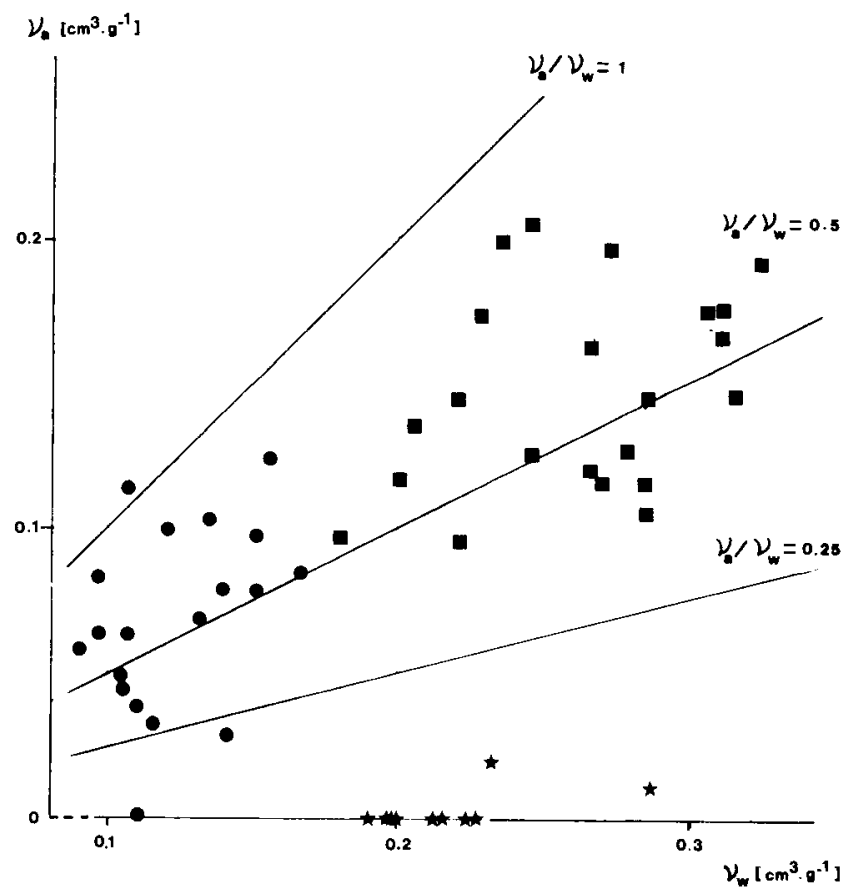

Figure 4

Volumes spécifiques d'air $\left(v_{d}\right)$ et d'eau $\left(v_{W}\right)$ d'agrégats prélevés dans la couche travaillée.

Air, $v_{a}$, and water, $v_{W}$, specific volumes in the deeper layer of the ploughed horizon.

- Mottes de 100 à $600 \mathrm{~cm}^{3}$ du traitement $C D$. 100 to $600 \mathrm{~cm}^{3}$ aggregates of the $C D$ plot.

- Mottes de 100 à $600 \mathrm{~cm}^{3}$ du traitement $C S$. 100 to $600 \mathrm{~cm}^{3}$ aggregates of the CS plot.

* Agrégats de 3 à $20 \mathrm{~cm}^{3}$ du traitement $C D$. 3 to $20 \mathrm{~cm}^{3}$ aggregates of the CD plot. s'accompagne donc d'une quasi-disparition de l'air intra-agrégat.

Le planimétrage des sections de couches travaillées indique que les rapports des surfaces entre plantation et état initial sont peu différents entre les traitements (1,05 pour CD et 1,08 pour $C S)$. Le calcul à partir de l'équation (4) montre que les volumes spécifiques d'air total sont élevés et peu différents entre les 2 traitements (tabl. 2).

TABLEAU 2

Bornage du volume spécifique d'air $\left(v_{a}\right)$ de la couche travaillée avant plantation (ep).

Air specific volume of ploughed layer before planting (ep).

\begin{tabular}{lcccc}
\hline \hline Traitements & \multicolumn{2}{c}{$\mathrm{CD}$} & \multicolumn{2}{c}{$\mathrm{CS}$} \\
\hline & $\begin{array}{c}v_{\mathrm{w}_{\mathrm{ep}}} \min \\
=0,10\end{array}$ & $\begin{array}{c}v_{\mathrm{w}_{\mathrm{ep}}} \max \\
=0,20\end{array}$ & $\begin{array}{c}v_{\mathrm{w}_{\mathrm{ep}}} \min \\
=0,20\end{array}$ & $\begin{array}{c}v_{\mathrm{w}_{\mathrm{ep}}} \max \\
=0,30\end{array}$ \\
\hline $\begin{array}{l}1 / \gamma_{\mathrm{d}_{\mathrm{e}}} \min \\
=0,94\end{array}$ & 0,42 & 0,32 & 0,38 & 0,28 \\
$\begin{array}{l}1 / \gamma_{\mathrm{d}} \max \\
=1,15\end{array}$ & 0,64 & 0,54 & 0,61 & 0,51 \\
\hline $\mathrm{S}_{\mathrm{ep}} / \mathrm{S}_{\mathrm{ei}}$ & \multicolumn{1}{c}{1,05} & \multicolumn{2}{c}{1,08} \\
\hline \hline
\end{tabular}

\section{Evolution des volumes spécifiques d'eau et d'air sous la culture irriguée (tabl. 3)}

Pour le traitement le plus arrosé (15), le volume spécifique d'air de la couche travaillée $(20-30 \mathrm{~cm})$ tend vers zéro $60 \mathrm{j}$ après la plantation ; pour les mêmes doses d'arrosage, ce volume reste élevé en CS, $100 \mathrm{j}$ après plantation.

Dans les 2 cas, les profils sont d'autant plus aérés que les doses d'irrigation sont plus faibles.

\section{B. Suivi du stockage d'eau par smectométrie dans les horizons profonds sous la culture irriguée (fig. 5)}

Le suivi du gonflement-retrait des horizons situés sous la couche travaillée montre un gonflement rapide en irrigation faible (I2), plus lent sous irrigation excédentaire (15). L'état reste ensuite stationnaire, sauf pour le traitement CS I2, pour lequel les horizons profonds subissent un retrait. L'événement pluvieux du $95^{\mathrm{e}} \mathrm{j}$ après la plantation $(54 \mathrm{~mm})$ provoque un gonflement important en dessous du fond de labour en CS I2, moindre en $\mathrm{CD}$ I2, et nul pour les traitements situés sous irrigation excédentaire (I5).

L'analyse du gonflement-retrait couche par couche révèle :

- en CD 12, l'absence de mouvement de la couche $40-60 \mathrm{~cm}$, alors que les couches $60-80$ et $80-120 \mathrm{~cm}$ gonflent sans délai puis restent stationnaires : la couche 40-60 est traversée, sans se réhumecter au point de gonfler ;

- en CS 12 , un phénomène identique mais avec un retrait de toutes les couches après le gonflement initial ;

- en CD I5, un gonflement rapide de la seule couche $40-60 \mathrm{~cm}$, les couches inférieures montrant un 
TABLEAU 3

Volumes spécifiques d'eau ( $\left.v_{w}\right)$ et d'air $\left(v_{\alpha}\right)$ en cours de culture $\left(\mathrm{cm}^{3} \cdot \mathrm{g}^{-1}\right)$. Water $\left(v_{w}\right)$ and air $\left(v_{o}\right)$ specific volumes during crop cycle $\left(\mathrm{cm}^{3} \cdot \mathrm{g}^{-1}\right)$.

\begin{tabular}{|c|c|c|c|c|c|c|c|c|c|c|c|c|c|c|c|c|c|}
\hline & \multirow{3}{*}{ I : } & \multicolumn{8}{|c|}{ C D } & \multicolumn{8}{|c|}{$\mathrm{Cs}$} \\
\hline & & \multicolumn{2}{|c|}{ I 2} & \multicolumn{2}{|c|}{ I 3} & \multicolumn{2}{|c|}{ I 4} & \multicolumn{2}{|c|}{ I 5} & \multicolumn{2}{|c|}{11} & \multicolumn{2}{|c|}{ I 2} & \multicolumn{2}{|c|}{ I 4} & \multicolumn{2}{|c|}{ I 5} \\
\hline & & $v_{w}$ & $v_{\mathrm{a}}$ & $v_{w}$ & $v_{a}$ & $v_{\mathrm{w}}$ & $v_{a}$ & $v_{w}$ & $v_{a}$ & $v_{w}$ & $v_{a}$ & $v_{w}$ & $v_{\mathrm{a}}$ & $v_{w}$ & $v_{\mathrm{a}}$ & $v_{w}$ & $v_{a}$ \\
\hline \multirow{3}{*}{$\mathrm{J}=60$} & $\underset{10-16}{Z}$ & 0,41 & 0,16 & 0,47 & 0,13 & & & 0,48 & 0,13 & & & 0,38 & 0,42 & & & 0,45 & 0,25 \\
\hline & $16-22$ & 0,41 & 0,22 & 0,47 & 0,22 & & & 0,49 & 0,10 & & & 0,39 & 0,40 & & & 0,45 & 0,40 \\
\hline & $22-27$ & 0,42 & 0,33 & 0,46 & 0,22 & & & 0,50 & 0,05 & & & 0,40 & 0,39 & & & 0,47 & 0,25 \\
\hline \multirow[t]{3}{*}{$\mathrm{J}=84$} & $8-16$ & 0,44 & 0,10 & 0,52 & 0,05 & & & 0,52 & 0,08 & 0,42 & 0,21 & & & & & & \\
\hline & $16-22$ & 0,43 & 0,26 & 0,50 & 0,14 & & & $\begin{array}{l}0,53 \\
0,53\end{array}$ & $\begin{array}{l}0,09 \\
0,09\end{array}$ & 0,41 & 0,24 & & & & & & \\
\hline & $22-28$ & 0,44 & 0,22 & 0,50 & 0,13 & & & $\begin{array}{l}0,52 \\
0,53 \\
0,56\end{array}$ & $\begin{array}{l}0,00 \\
0,00 \\
0,00\end{array}$ & 0,32 & 0,31 & & & 0,50 & 0,33 & 0,53 & 0,28 \\
\hline $\mathbf{J}=100$ & $22-28$ & & & & & 0,52 & 0,19 & 0,56 & 0,00 & & & & & & & 0,52 & 0,14 \\
\hline
\end{tabular}

$\mathrm{J} \quad$ : Jours après plantation. Days after planting.

$\mathrm{Z}$ : Profondeur, $\mathrm{cm}$. Depth.

$\mathrm{CD}$ : Charrue à disques. Disc plough.

CS : Charrue à socs. Plough.

I : Traitements d'irrigation. Irrigation treatments.

retard au gonflement d'autant plus long qu'elles sont profondes ;

- en CS 15 enfin, un gonflement plus rapide en 80$120 \mathrm{~cm}$ que dans le traitement précédent ; la couche 4060 gonfle plus progressivement, $60-80 \mathrm{~cm}$ ne bouge pas.

\section{Résultats sur la culture}

L'analyse des moyennes du nombre cumulé de fleurs nouées pour chaque traitement de préparation du sol, tous traitements d'irrigation confondus, montre qu'elles ne sont pas significativement différentes. Pour chacun des traitements de préparation du sol, le classement des traitements d'irrigation est identique : le nombre de fleurs nouées produites décroît avec l'augmentation des doses d'irrigation. Cependant, la divergence entre les courbes d'évolution de ce paramètre en fonction du temps est plus ou moins précoce et marquée suivant l'état structural (fig. 6). En CS, les moyennes ne sont jamais significativement différentes alors qu'elles le sont en CD dès le $54^{\mathrm{e}} \mathrm{j}$ au seuil 1 p. 100 . A partir de cette date, le traitement I5 en CD ne produit plus de fruits.

L'analyse du rendement final (nombre de fruits total par plante) en fonction de la pluviométrie montre des pentes négatives dans les 2 cas, mais plus forte en CD qu'en CS (fig. 7).

L'examen de l'état du système racinaire à la floraison montre des taches de couleur rouille autour des racines proches du fond de labour en CD I5 seulement. Le système racinaire s'arrête alors à ce niveau, alors qu'il descend plus bas dans les autres traitements. En fin de culture, les racines se développent jusqu'à $100 \mathrm{~cm}$ en $\mathrm{CS} \mathrm{I} 2$, et jusqu'à $70 \mathrm{~cm}$ en $\mathrm{CD} \mathrm{I} 2$; à l'inverse, dans les traitements I5, l'essentiel de l'enracinement est demeuré dans la couche travaillée.

\section{DISCUSSION}

1. La désagrégation des mottes en surface, sous l'action du climat et le granoclassement lié aux passages successifs d'outils provoquent l'enfouissement de terre fine très sèche sur le fond de labour

La figure 8 retrace l'évolution des volumes spécifiques d'air et d'eau, sous l'astion des outils et du climat avant la plantation, puis au fond de la couche travaillée sous les différents traitements d'irrigation. Par rapport à l'état initial, le labour provoque une augmentation globale de porosité pour l'air, analogue pour les 2 préparations. La différence réside essentiellement dans la distribution de cette porosité.

L'état structural CD est caractérisé à la plantation par un arrangement d'agrégats saturés, malgré leur faible teneur en eau, de volumes variables en haut de la couche travaillée, de dimensions centi- à millimétriques au fond du labour. En effet, après le premier labour, les mottes se désagrègent en surface sous l'action des alternances d'humectations et dessiccations, pour donner un mulch d'agrégats millimétriques de quelques centimètres ; ce dernier retarde la dessiccation, et donc l'évolution des mottes sous jacentes. Le second passage, en remontant ces mottes qui finissent alors de se désagréger, épand les agrégats fins et secs sur le fond de labour. Le volume spécifique d'air total augmente ou reste celui de l'état initial, mais celui des agrégats de 3 à $20 \mathrm{~cm}^{3}$ tend à s'annuler; il en est a fortiori de même pour les agrégats constituant la terre fine. Cette 

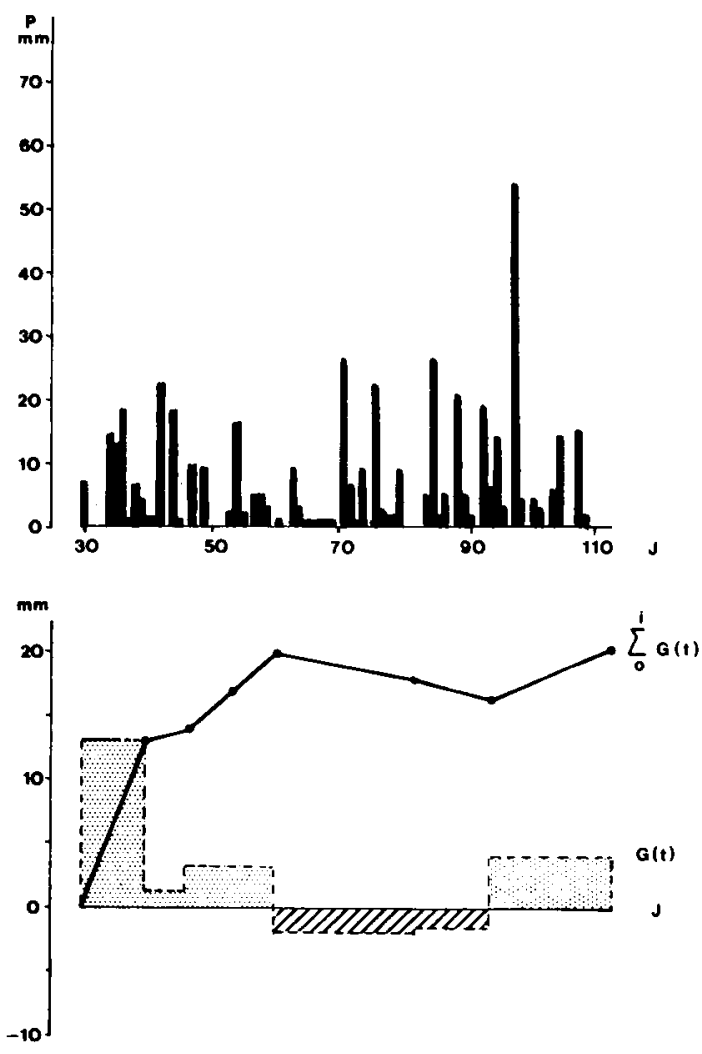

B

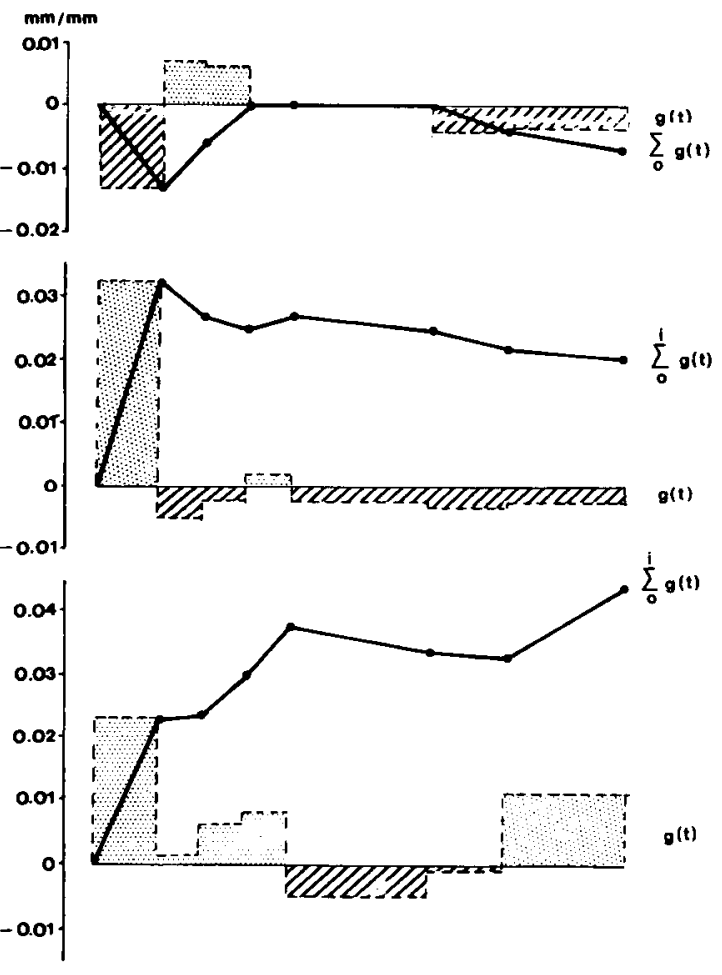

CD
A
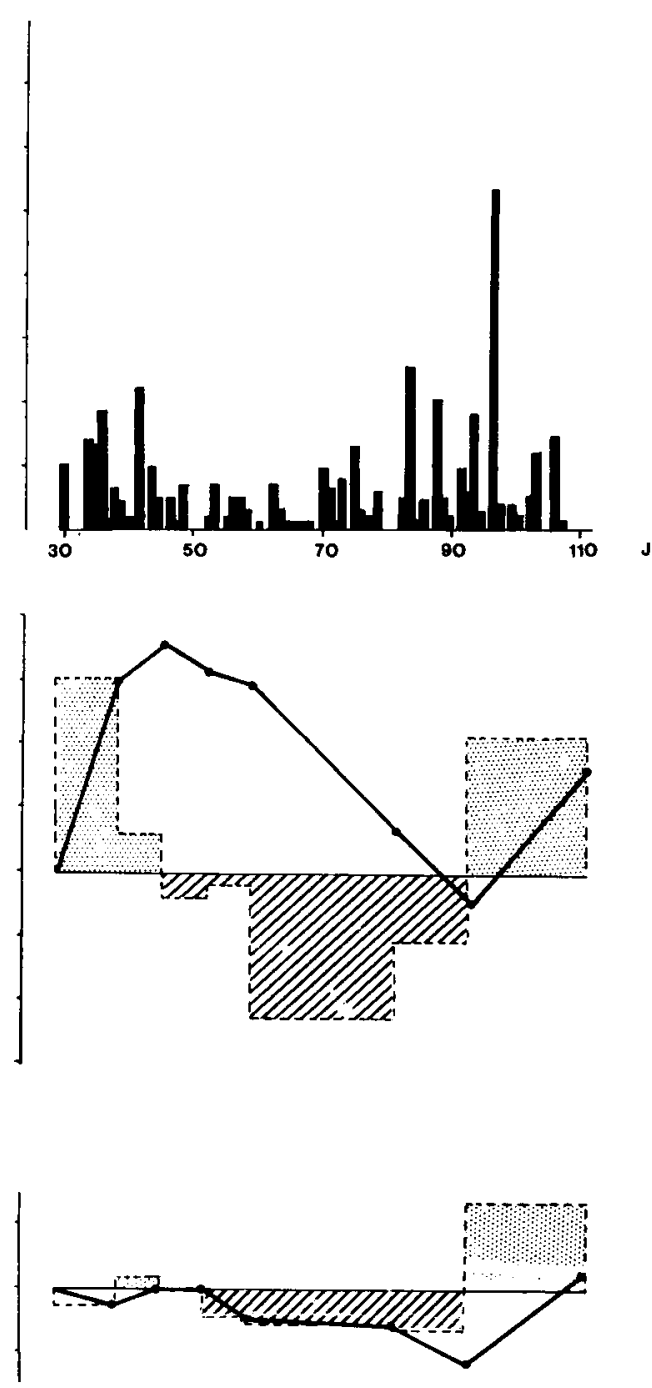

C

D

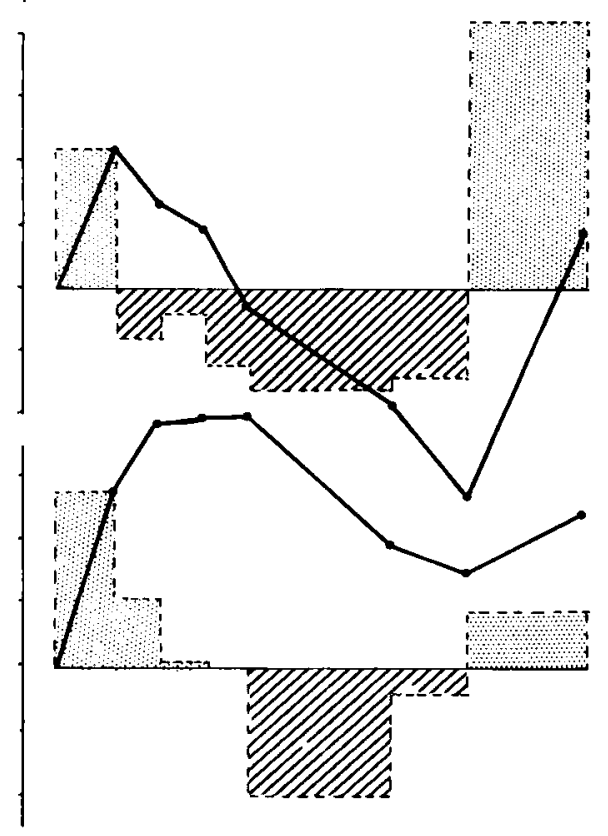

Figure 5

Evolution du gonflement-retrait des horizons situés sous le fond de labour.

Swelling and shrinking of the deeper layers.

$P \quad$ Pluviométrie. Rainfall.

$J \quad$ Nombre de jours après plantations. Days after planting.
CS

12

$\sum^{\mathrm{i}} \mathrm{G}(\mathrm{t})$ Gonflement cumulé à $40 \mathrm{~cm}$ depuis la pose. Cumulated swelling at $40 \mathrm{~cm}$ depth: A.

$G(t)$ Gonflement cumulé entre 2 dates à $40 \mathrm{~cm}$. Cumulated swelling at $40 \mathrm{~cm}$ between two dates: $A$. Gonflement. Swelling. Retrait. Shrinking. 

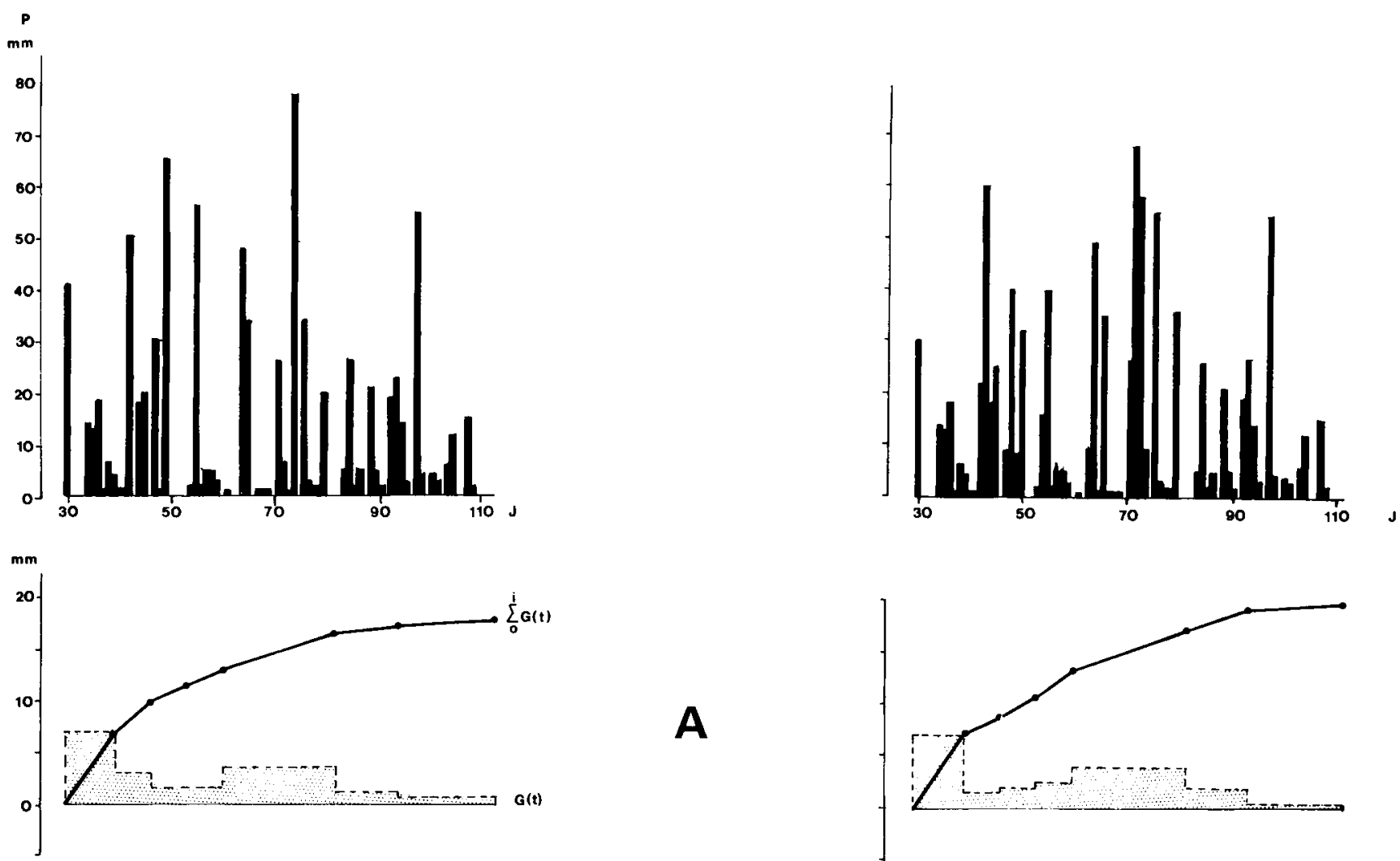

A

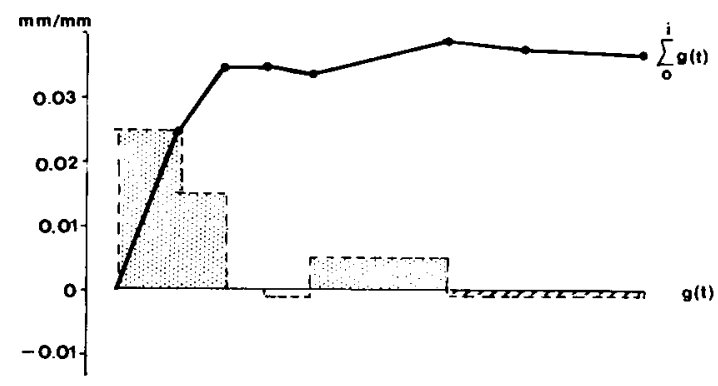

B
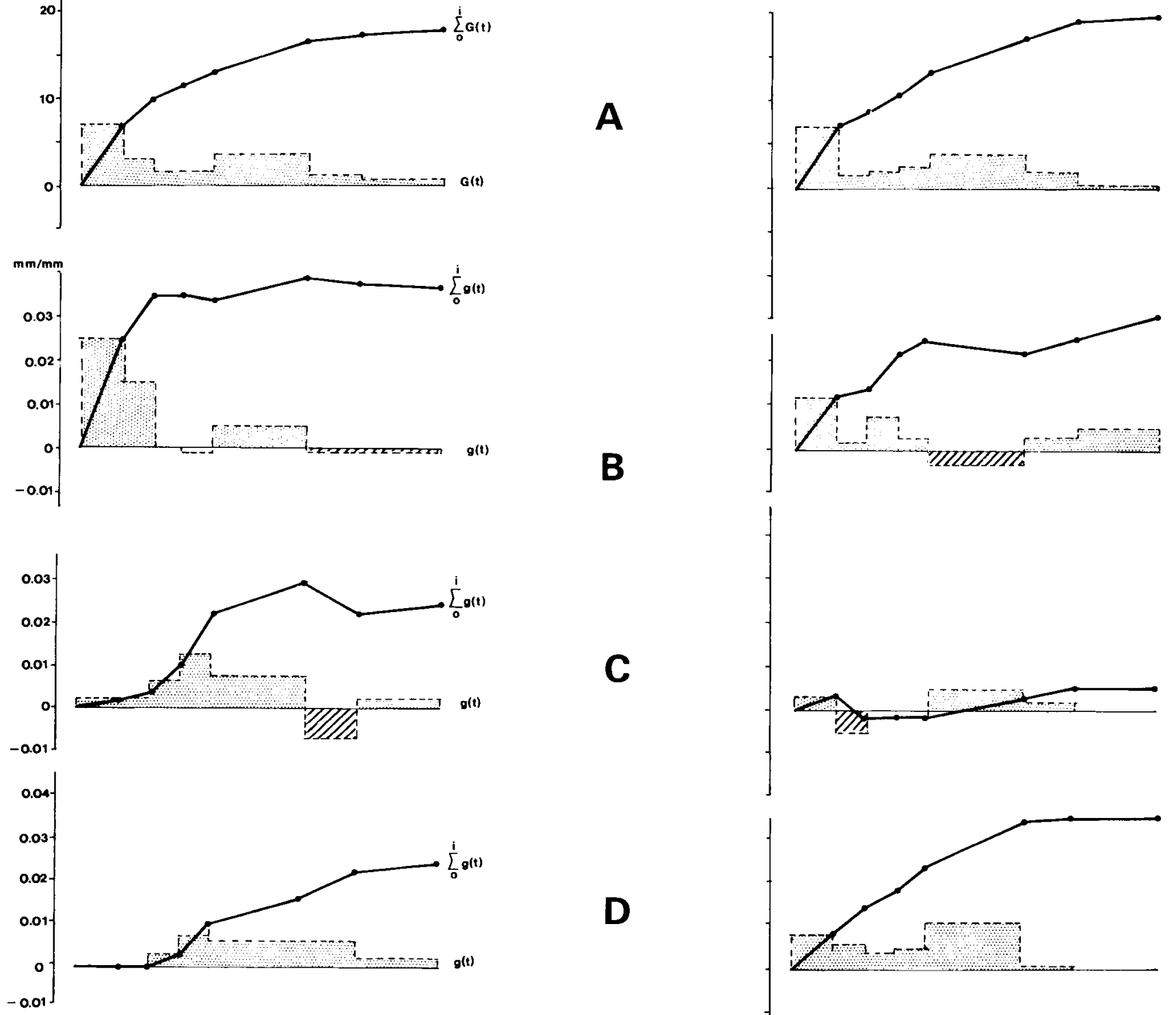

C

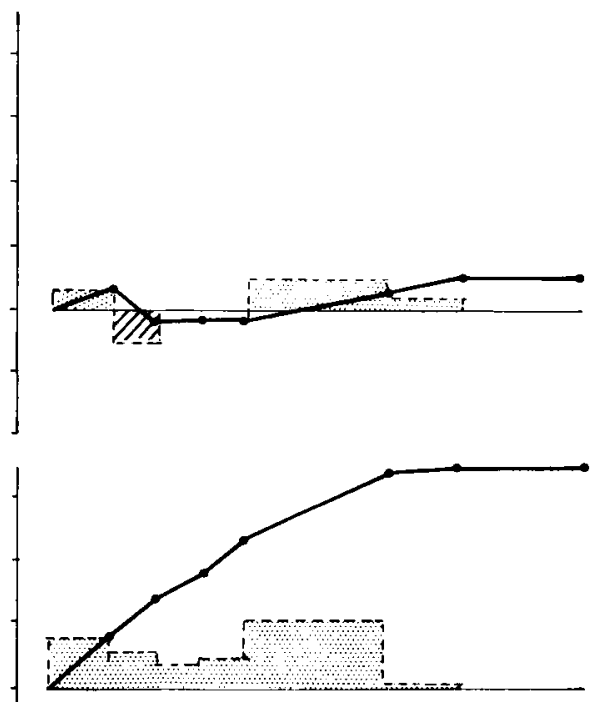

D

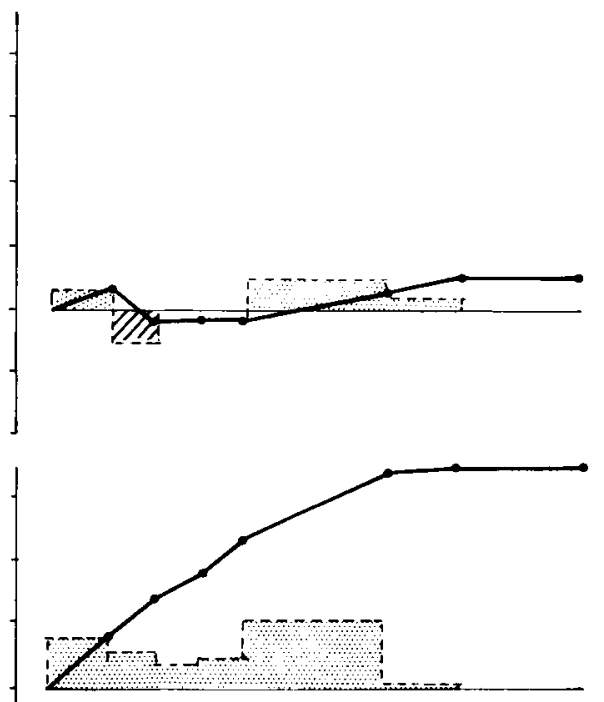

CD

15

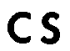

Figure 5 (suite)

$\sum_{0}^{\mathrm{i}} \mathrm{g}(\mathrm{t})$ Gonflement élémentaire de la couche depuis la pose. Elementary swelling of the layer.

$g(t) \quad$ Gonflement élementaire entre 2 dates de la couche. Elementary swelling between two dates of the layer.

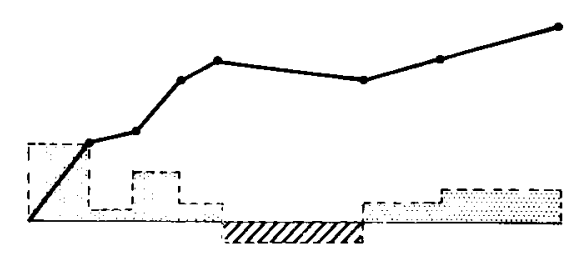

$B: 40-60 \mathrm{~cm}$

$C: 60-80 \mathrm{~cm}$

$D: 80-120 \mathrm{~cm}$.

$C D \quad$ Charrue à disques. Disc plough.

CS Charrue à socs. Share plough. 
TABLEAU $4 a$

Moyenne du nombre de fleurs nouées par plante et par date, intervalle de confiance de la moyenne (95\%) et résultats des tests de Bartlett et analyse de variance (charrue à disques).

Mean fruit set at each date, LSD (5\%), Bartlett test and anova (disc plough).

\begin{tabular}{|c|c|c|c|c|c|c|c|c|}
\hline I & I 1 & 12 & I 3 & I 4 & I 5 & Moy. & $\begin{array}{c}\text { Bartlett } \\
\mathrm{P}(\mathrm{BT}<\mathrm{BTobs})\end{array}$ & $\begin{array}{c}\text { Anova } \\
\mathrm{P}(\mathrm{F}<\text { Fobs })\end{array}$ \\
\hline $\mathrm{N}$ & 17 & 9 & 9 & 11 & 8 & & & \\
\hline \multirow[t]{2}{*}{$\mathbf{J}=30$} & 3,6 & 3,8 & 3,6 & 2,4 & 3,8 & 3,6 & \multirow{2}{*}{ - } & \multirow{2}{*}{-} \\
\hline & 0,6 & 0,7 & 1,1 & 1,3 & 1,2 & & & \\
\hline \multirow[t]{2}{*}{$\mathrm{J}=40$} & 7,1 & 6,8 & 8,3 & 7,9 & 7,4 & 7,5 & \multirow{2}{*}{-} & \multirow{2}{*}{-} \\
\hline & 0,8 & 1,1 & 1,7 & 1,8 & 1,6 & & & \\
\hline$J=48$ & $\begin{array}{r}13,1 \\
1,1\end{array}$ & $\begin{array}{r}14,0 \\
2,6\end{array}$ & $\begin{array}{r}14,0 \\
2,5\end{array}$ & $\begin{array}{r}10,9 \\
1,9\end{array}$ & $\begin{array}{r}10,9 \\
1,6\end{array}$ & 12,6 & $\begin{array}{l}0,739 \\
\text { NS }\end{array}$ & $\begin{array}{c}0,927 \\
\text { NS }\end{array}$ \\
\hline $\mathrm{J}=54$ & $\begin{array}{r}17,6 \\
1,6\end{array}$ & $\begin{array}{r}17,6 \\
3,3\end{array}$ & $\begin{array}{r}15,4 \\
2,4\end{array}$ & $\begin{array}{r}12,2 \\
1,8\end{array}$ & $\begin{array}{r}11,4 \\
1,8\end{array}$ & 15,2 & $\begin{array}{c}0,661 \\
\text { NS }\end{array}$ & $\begin{array}{c}0,999 \\
\text { NS }\end{array}$ \\
\hline $\mathrm{J}=61$ & $\begin{array}{r}19,5 \\
1,9\end{array}$ & $\begin{array}{r}19,0 \\
3,0\end{array}$ & $\begin{array}{r}16,7 \\
1,4\end{array}$ & $\begin{array}{r}13,6 \\
1,5\end{array}$ & $\begin{array}{r}11,5 \\
1,9\end{array}$ & 16,6 & $\begin{array}{c}0,653 \\
\text { NS }\end{array}$ & $\begin{array}{c}0,999 \\
\text { NS }\end{array}$ \\
\hline $\mathrm{J}=68$ & $\begin{array}{r}19,8 \\
2,2\end{array}$ & $\begin{array}{r}20,6 \\
3,4\end{array}$ & $\begin{array}{r}17,2 \\
3,0\end{array}$ & $\begin{array}{r}14,1 \\
1,7\end{array}$ & $\begin{array}{r}11,5 \\
1,9\end{array}$ & 17,1 & $\begin{array}{c}0,751 \\
\text { NS }\end{array}$ & $\begin{array}{c}0,999 \\
\text { NS }\end{array}$ \\
\hline $\mathrm{J}=75$ & $\begin{array}{r}19,9 \\
2,2\end{array}$ & $\begin{array}{r}20,7 \\
3,4\end{array}$ & $\begin{array}{r}17,2 \\
3,0\end{array}$ & $\begin{array}{r}14,1 \\
1,7\end{array}$ & $\begin{array}{r}11,5 \\
1,9\end{array}$ & 17,2 & $\begin{array}{c}0,770 \\
\text { NS }\end{array}$ & $\begin{array}{c}0,999 \\
\text { NS }\end{array}$ \\
\hline $\mathrm{J}=83$ & $\begin{array}{r}20,0 \\
2,3\end{array}$ & $\begin{array}{r}20,7 \\
3,4\end{array}$ & $\begin{array}{r}17,2 \\
3,0\end{array}$ & $\begin{array}{r}14,1 \\
1,7\end{array}$ & $\begin{array}{r}11,5 \\
1,9\end{array}$ & 17,2 & $\begin{array}{c}0,772 \\
\text { NS }\end{array}$ & $\begin{array}{c}0,999 \\
\text { NS }\end{array}$ \\
\hline
\end{tabular}

I : Traitements d'irrigation. Irrigation treatments.

$\mathrm{J}:$ Nombre de jours après plantation. Days after planting.

$\mathrm{n}$ : Effectif. Plant number.

perte d'air intra-agrégat exprime la rupture préférentielle des mottes au niveau des zones fragiles que représentent les pores de grande taille (fissures, tubules, racines en décomposition). Cette couche, sur le fond de labour présente par son degré de dessèchement un fort gonflement potentiel et une porosité pour l'air exclusivement intersticielle.

Dans la couche travaillée du traitement CS, on constate une conservation de l'air intra-motte par rapport à l'état initial, et une augmentation de l'aération globale grâce à l'air inter-mottes introduit par le labour, qui demeure important à la plantation. Le gonflement potentiel des mottes est plus faible qu'en CD puisqu'elles sont plus humides.

\section{Le fond de labour entrave l'infiltration profonde lorsque les fentes sont oblitérées par les passages d'outils}

Sous irrigation rationnée (I2), l'absence de gonflement de la couche $40-60 \mathrm{~cm}$ traduit le passage rapide de l'eau en profondeur, par les fentes restées fonctionnelles. La réhumectation intéresse les voisinages du fond de ces fentes, soit $60-80$ et surtout $80-120 \mathrm{~cm}$. Pour le traitement CS I2, 60-80 et de façon moindre
80-120 cm se rétractent. L'évaporation du sol nu ne peut être mise en cause pour expliquer un dessèchement des horizons profonds : celle-ci s'annule rapidement entre 2 irrigations par la formation du mulch de surface (RITCHIE \& BURNETT, 1971) ; compte tenu de la faible vitesse de diffusion de l'eau dans l'argile continue, l'affaissement constaté est donc la traduction d'une extraction racinaire en profondeur, compatible avec les profils racinaires observés en fin de culture.

Les comportements hydriques associés aux 2 états structuraux $C D$ et $C S$ sont, en revanche, différents sous irrigation excédentaire. En $\mathrm{CD}$, le remplissage affectant immédiatement la couche $40-60 \mathrm{~cm}$, sans concerner les couches inférieures, traduit le blocage précoce de l'infiltration per-fissurale rapide. Seule subsiste la lente diffusion intramassif. Dans le même temps, une nappe perchée est observable immédiatement au-dessus du fond de labour ou subsistent les lissages.

En CS I5, le gonflement de la couche $40-60 \mathrm{~cm}$, plus lent, s'accompagne d'un gonflement de la couche 80 120 ; l'infiltration profonde n'est donc pas bloquée, elle se fait probablement de manière très localisée, et donc surtout par les fissures, puisque $60-80 \mathrm{~cm}$, ne gonflant pas, est traversée sans être réhumectée. 
TABLEAU $4 b$

Moyenne du nombre de fleurs nouées par plante et par date, intervalle de confiance de la moyenne (95\%) et résultats des tests de Bartlett et analyse de variance (charrue à socs).

Mean fruit set at each date, LSD (5\%), Bartlett test and anova (share plough).

\begin{tabular}{|c|c|c|c|c|c|c|c|c|}
\hline I & I 1 & I 2 & I 3 & I 4 & I 5 & Moy. & $\begin{array}{c}\text { Bartlett } \\
\mathrm{P}(\mathrm{BT}<\mathrm{BTobs})\end{array}$ & $\begin{array}{c}\text { Anova } \\
\mathrm{P}(\mathrm{F}<\text { Fobs })\end{array}$ \\
\hline $\mathrm{N}$ & 14 & 9 & 6 & 6 & 5 & & & \\
\hline \multirow[t]{2}{*}{$\mathrm{J}=30$} & 3,1 & 3,7 & 3,7 & 2,7 & 4,4 & 3,4 & \multirow{2}{*}{-} & \multirow{2}{*}{-} \\
\hline & 0,9 & 1,2 & 1,8 & 1,9 & 2,6 & & & \\
\hline \multirow[t]{2}{*}{$J=40$} & 5,9 & 7,7 & 8,7 & 6,5 & 9,2 & 7,2 & \multirow{2}{*}{-} & \multirow{2}{*}{-} \\
\hline & 1,3 & 1,6 & 2,1 & 2,1 & 2,3 & & & \\
\hline $\mathbf{J}=48$ & $\begin{array}{r}11,6 \\
1,4\end{array}$ & $\begin{array}{r}14,3 \\
3,5\end{array}$ & $\begin{array}{r}13,8 \\
3,1\end{array}$ & $\begin{array}{r}12,3 \\
2,0\end{array}$ & $\begin{array}{r}12,2 \\
1,6\end{array}$ & 12,7 & $\begin{array}{c}0,944 \\
\text { NS }\end{array}$ & $\begin{array}{c}0,579 \\
\mathrm{NS}\end{array}$ \\
\hline $\mathrm{J}=54$ & $\begin{array}{r}15,1 \\
1,7\end{array}$ & $\begin{array}{r}17,1 \\
3,8\end{array}$ & $\begin{array}{r}17,7 \\
4,3\end{array}$ & $\begin{array}{r}15,0 \\
2,1\end{array}$ & $\begin{array}{r}13,0 \\
1,4\end{array}$ & 15,7 & $\begin{array}{l}0,965 \\
\text { NS }\end{array}$ & $\begin{array}{c}0,687 \\
\text { NS }\end{array}$ \\
\hline$J=61$ & $\begin{array}{r}18,5 \\
1,8\end{array}$ & $\begin{array}{r}19,1 \\
3,7\end{array}$ & $\begin{array}{r}19,8 \\
3,5\end{array}$ & $\begin{array}{r}16,0 \\
2,7\end{array}$ & $\begin{array}{r}15,6 \\
1,7\end{array}$ & 18,1 & $\begin{array}{l}0,744 \\
\text { NS }\end{array}$ & $\begin{array}{l}0,708 \\
\text { NS }\end{array}$ \\
\hline$J=68$ & $\begin{array}{r}20,1 \\
2,5\end{array}$ & $\begin{array}{r}20,1 \\
3,7\end{array}$ & $\begin{array}{r}19,8 \\
3,5\end{array}$ & $\begin{array}{r}16,3 \\
2,7\end{array}$ & $\begin{array}{r}15,8 \\
1,9\end{array}$ & 18,9 & $\begin{array}{l}0,661 \\
\mathrm{NS}\end{array}$ & $\stackrel{0,814}{\mathrm{NS}}$ \\
\hline $\mathrm{J}=75$ & $\begin{array}{r}20,6 \\
2,6\end{array}$ & $\begin{array}{r}20,2 \\
3,2\end{array}$ & $\begin{array}{r}19,8 \\
3,5\end{array}$ & $\begin{array}{r}17,0 \\
3,0\end{array}$ & $\begin{array}{r}16,0 \\
1,5\end{array}$ & 19,3 & $\begin{array}{c}0,699 \\
\text { NS }\end{array}$ & $\begin{array}{c}0,782 \\
\text { NS }\end{array}$ \\
\hline $\mathrm{J}=83$ & $\begin{array}{r}20,6 \\
2,6\end{array}$ & $\begin{array}{r}20,2 \\
3,2\end{array}$ & $\begin{array}{r}19,8 \\
3,5\end{array}$ & $\begin{array}{r}17,0 \\
3,0\end{array}$ & $\begin{array}{r}16,0 \\
1,5\end{array}$ & 19,3 & $\begin{array}{c}0,699 \\
\text { NS }\end{array}$ & $\begin{array}{c}0,782 \\
\text { NS }\end{array}$ \\
\hline
\end{tabular}

3. La capacité de gonflement de la terre fine, faite d'agrégats saturés tout en étant très secs, enfouie au contact du fond de labour, provoque la formation d'un milieu continu et saturé sous irrigation excédentaire

Le volume spécifique d'air diminue rapidement dans la base de la couche travaillée en $C D$, sous irrigation excédentaire (fig. 8). L'eau qui ne peut s'infiltrer vers les couches profondes provoque le gonflement des agrégats saturés initialement très secs, qui deviennent coalescents au détriment de la porosité intersticielle. Il en résulte la formation d'une couche continue, de faible épaisseur.

En CS, en revanche, le volume spécifique d'air reste élevé. L'eau peut s'infiltrer par les fentes de retrait ; la saturation de la base de la couche travaillée ne peut apparaître que tardivement, lorsque la réhumectation du fond de labour a été suffisante pour que celles-ci soient refermées (état saturé de l'horizon). Le fonctionnement hydrique du sol non travaillé se rapproche de celui observé en conditions naturelles (JAILLARD \& CABIDOCHE, 1984).

La production de fleurs nouées en fonction du temps traduit ce phénomène. Les traitements d'irrigation se différencient plus largement et plus tôt en CD qu'en CS. L'effet dépressif sur le rendement final associé aux irrigations excédentaires apparaît beaucoup plus mar- qué en $\mathrm{CD}$. Par delà la saturation, les gaines rouilles périracinaires observées en CD I5 témoignent de l'apparition d'un niveau réducteur ; le développement et le fonctionnement du système racinaire sont alors perturbés : circonscrit aux 20 premiers centimètres, ce dernier ne peut extraire les besoins en eau de la plante que si les irrigations sont très rapprochées. Notre essai ne nous ayant pas permis de jouer sur les fréquences, un certain déficit hydrique a pu apparaître en cours de culture. En milieu paysan, la gestion correcte des irrigations à fréquence élevée suppose une vigilance extrême sur les doses, qui est rarement réalisée. Ainsi le phénomène d'excès d'eau s'amplifie au cours de la culture.

\section{CONCLUSION}

Dans les vertisols, on cherche généralement à obtenir une structure fine, afin de favoriser l'extraction racinaire dans un milieu initialement continu, à diffusion lente de l'eau.

La solution la moins onéreuse pour obtenir cette structure fine consiste à désagréger la totalité de la couche travaillée en favorisant les alternances de dessiccations et d'humectations. 


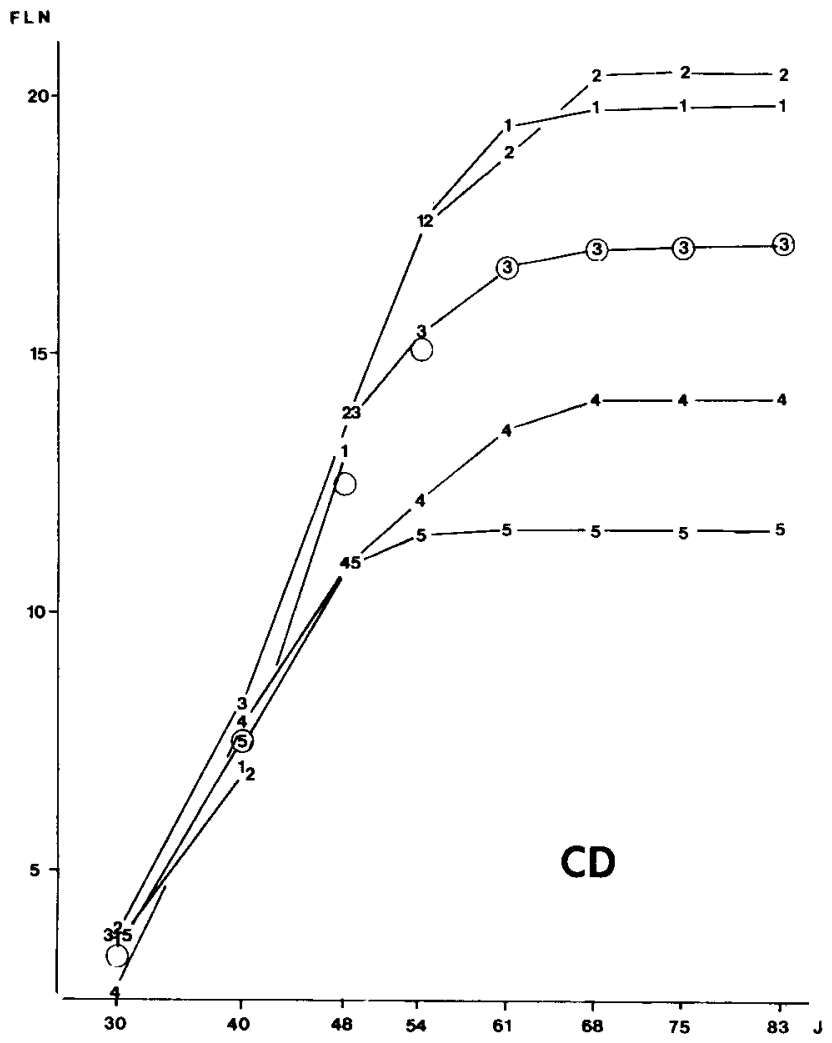

Figure 6

Evolution du nombre cumulé moyen de fleurs nouées produites par plantes par dates.

Cumulative average fruit set.

$J \quad$ Jours après plantation. Days after planting.

FLN Nombre de fleurs nouées. Fruit set.

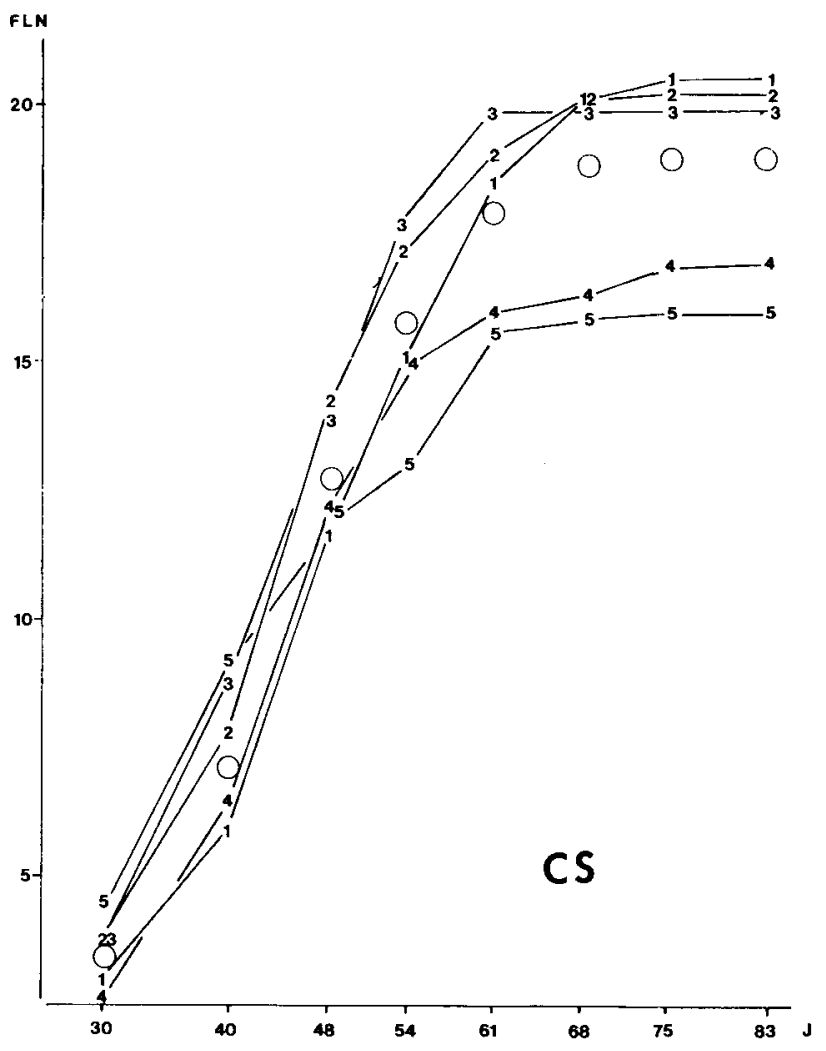

Moyenne par traitement de préparation du sol. Average for the two tillage treatments.

Traitement II. II treatment.

Traitement 12. I2 treatment.

Traitement 13. 13 treatment.

Traitement 14. I4 treatment.

Traitement 15 . I5 treatment.

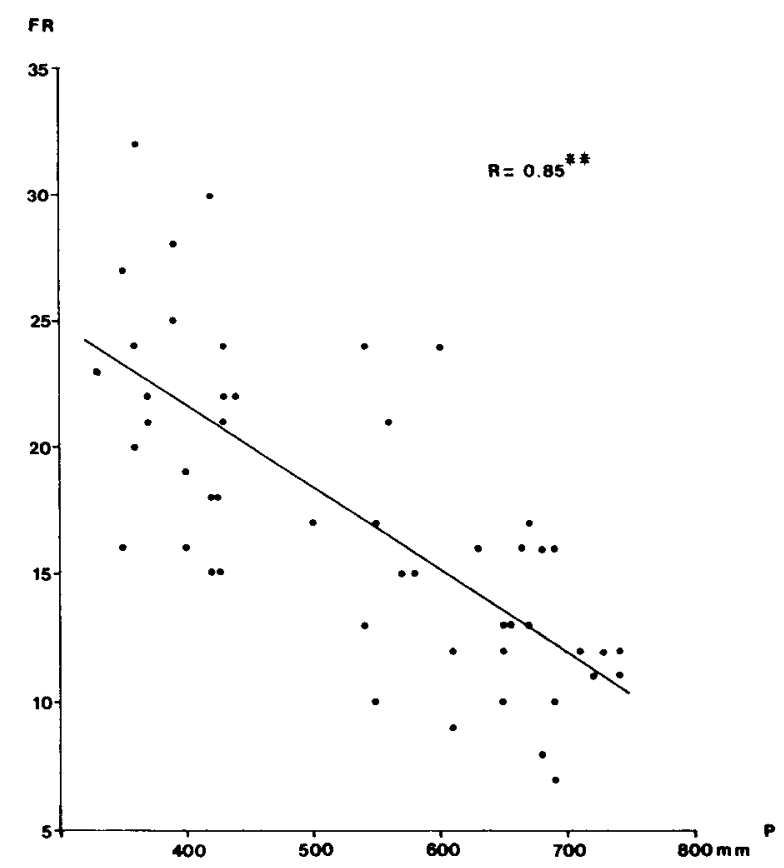

CD

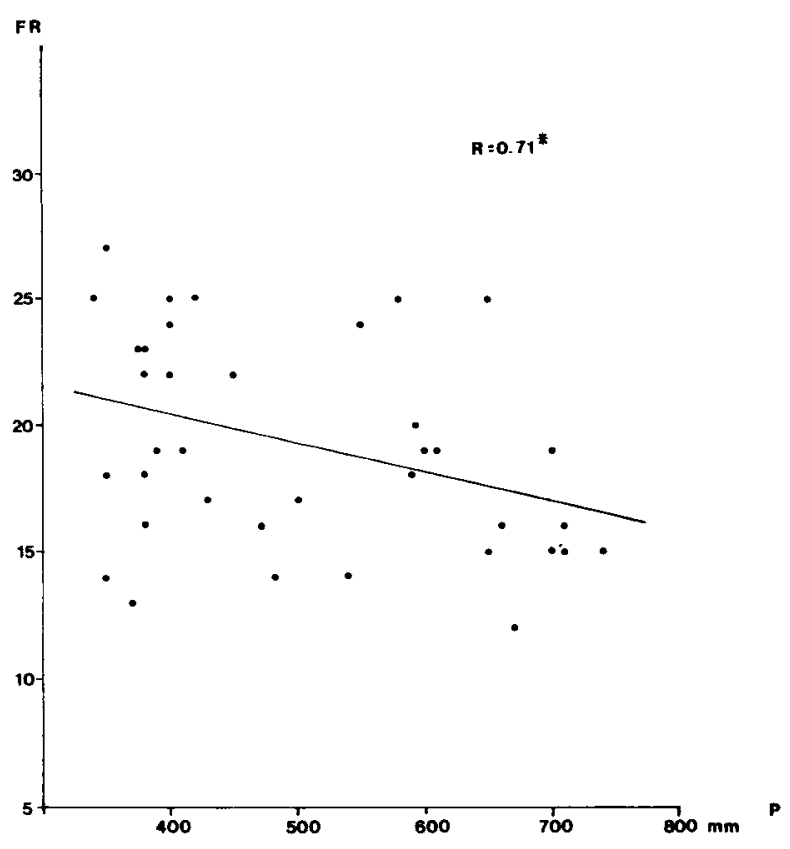

CS

Figure 7

Nombre de fruits produits par plante et dose d'irrigation totale. Fruit number and irrigation dose.

$P \quad$ Dose d'arrosage reçue par la plante. Irrigation dose. FR Nombre de fruits produits. Fruit number. 
$\left(\mathrm{cm}^{3} \cdot \mathrm{g}^{-1}\right)$

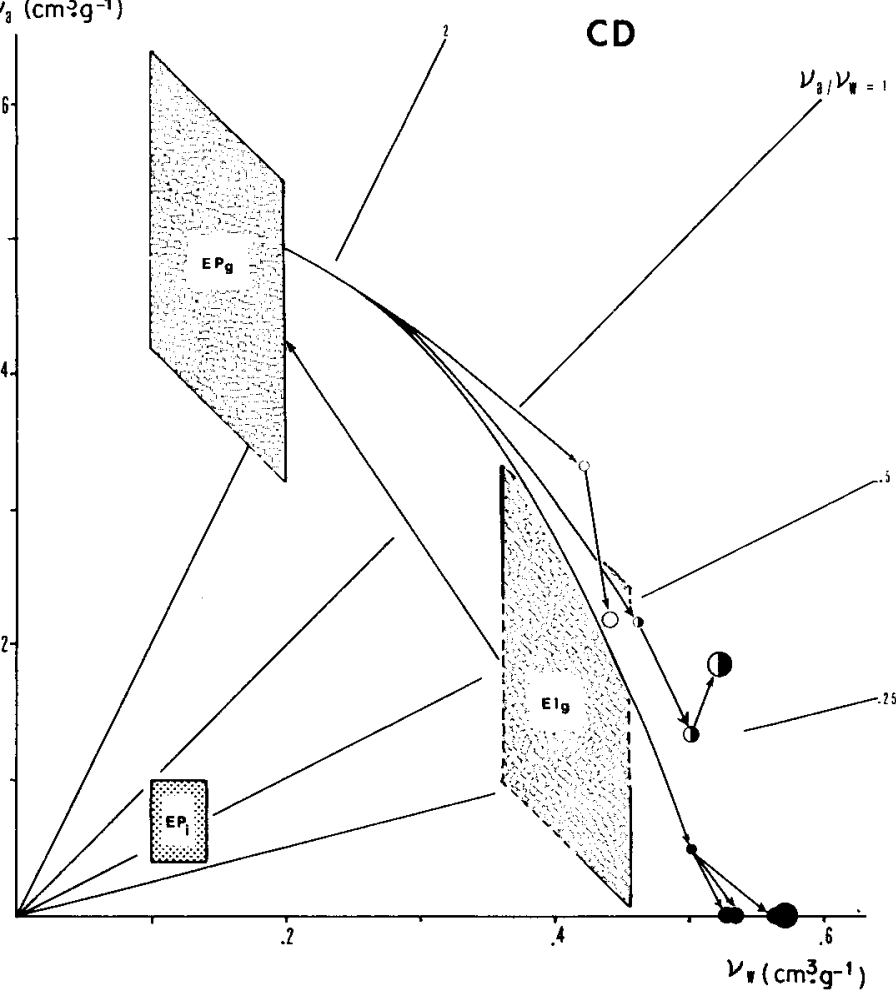

Figure 8

Trajectoire d'évolution des volumes massiques d'air ( $\left.v_{\alpha}\right)$ et d'eau $\left(v_{w}\right)$, sous l'action des outils et du climat, puis sous culture de tomate pour les différents traitements d'irrigation.

Pattern of change of the air $\left(\nu_{a}\right)$ and water $\left(\nu_{W}\right)$ specific volumes.

$C D$ Charrues à disques. Disc plough.

CS Charrue à soc. Plough.

EIg Couche 0-30 cm avant travail. $0-30 \mathrm{~cm}$ layer before tillage.

EPg Couche travaillée avant la plantation. Tilled layer before planting.

EPi Intramotte avant la plantation. Intra-clod before planting.

A cause de la formation d'un mulch, bloquant le dessèchement du fond de la couche travaillée une première fois, un deuxième passage d'outils est généralement nécessaire. L'état structural, en fin d'itinéraire de préparation du sol, est constitué d'agrégats centi- à millimétriques, très secs et contenant peu d'air.

Cet état structural s'accompagne d'une oblitération des fentes de retrait sous-jacentes par des lissages, consécutifs aux passages répétés d'outils tels que la charrue à disques ; on assiste alors à un blocage du drainage, qui provoque le regonflement avec prise en masse des agrégats à la base de la couche travaillée, qui se sature rapidement.

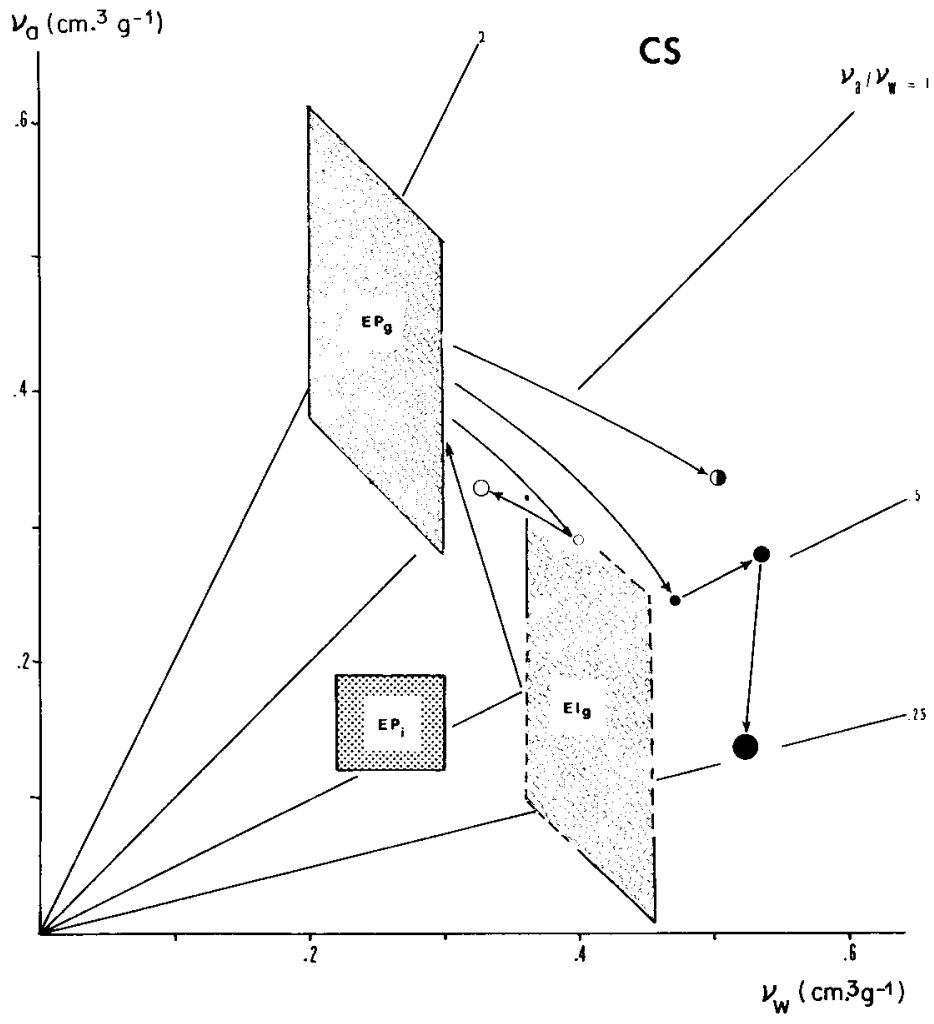

\begin{tabular}{|c|c|c|c|}
\hline $\begin{array}{l}\text { Couche travaillée, } 20-30 \mathrm{~cm} \\
\text { Tilled layer, } 20-30 \mathrm{~cm}\end{array}$ & $I I, I 2$ & $13, I 4$ & 15 \\
\hline $\begin{array}{l}60 \mathrm{j} \text { après plantation } \\
60 \text { days after planting }\end{array}$ & 0 & - & $\bullet$ \\
\hline $\begin{array}{l}84 j \text { après plantation } \\
84 \text { days after planting }\end{array}$ & 0 & - & $\bullet$ \\
\hline $\begin{array}{l}100 \mathrm{j} \text { après plantation } \\
100 \text { days after planting }\end{array}$ & & 0 & \\
\hline
\end{tabular}

Cette couche à haute susceptibilité de saturation, qui peut avoir toute son utilité en tant que réserve en eau pour une phase d'implantation de cultures sous pluviométrie discontinue (par exemple plantation de la canne à sucre par boutures), devient un handicap pour des cultures irriguées dans des conditions où les doses sont mal maîtrisées.

Dans ce dernier cas, on recherchera un outil respectant le système de porosité du sol non travaillé à la limite de la couche travaillée, quitte à ce que l'état structural soit plus grossier.

Reçu le 29 juillet 1986. Accepté le 6 janvier 1987.

\section{RÉFÉRENCES BIBLIOGRAPHIQUES}

Cabidoche Y. M., 1986. Distribution des sols à argile gonflante sur calcaires récifaux (zone Caraibes). Utilisation de la résistivité électrique. Premier séminaire scientifique de Pédologie pour la région de l'Amérique centrale et des Caraibes. Sol et Eau, 8-20 avril 1985, Ed. ORSTOM, pp. 187-219.

Cabidoche Y. M., Jaillard B., Ney B., 1986. Dynamique de l'eau dans les vertisols sur calcaires récifaux. Premier séminaire scientifique de Pédologie pour la région de l'Amérique centrale et des Caraibes. Sol et Eau, 8-20 avril 1985. Ed. ORSTOM, pp. 449-478.
Cavicchi S., Silvetti E., 1976. Yield in tomato. I. - Multiple regression between yield and yield components. Genet. agr., 30 (3/4), pp. 293-313.

Duthion C., 1973. Etude des effets d'un excès d'eau sur les plantes. Exemple de la tomate. C. R. Acad. Sci., pp. 1461-1468.

Flocker W. J., Menary R. C., 1960. Some physiological responses in two tomatoes varieties associated with level of soil bulk densities. $\mathrm{Hil}$ degardia, 30, pp. 101-121. 
Jaillard B., Cabidoche Y. M., 1984. Etude de la dynamique de l'eau dans un sol argileux gonflant : dynamique hydrique. Sci. Sol, $\mathrm{n}^{\circ} 3$, pp. 239-251.

Ney B., 1987. Fonctionnement hydrique de sols à argile gonflante cultivés. I. - Analyse des influences du travail du sol et de l'irrigation sur le fonctionnement hydrique de sols à argile gonflante dans des exploitations de Guadeloupe (F.W.I.). Agronomie, 7 (4), 247 256.

Perigaud S., 1967. Effet du manque d'oxygène et de l'excès d'eau au niveau des racines sur la croissance et la nutrition globale de la plante. Ann. agron., 18 (5), pp. 485-506.
Ritchie J. T., Burnett E., 1971. Dryland evaporative flux in subhumid climate. II. - Plant influences. Agron. J., 63 (1), pp. 56-61.

Schoch P. G., De Villele O., Granier J., 1975. Besoins climatiques en eau des cultures aux Antilles françaises. Nouv. agron. Antilles Guyane, 1-2, pp. 101-108.

Singh R. R., Singh M. N., 1980. Correlation studies in tomato. Indian J. Agric. Sci., 60 (8), pp. 595-598.

Wiebe H., 1949. Soil aeration in relation to growth of tomatoes. Iowa Acad. Sci. Proc., 59, 183-188. 